\title{
Quasilinear irreversible thermodynamics of a low-temperature-differential kinematic Stirling heat engine
}

\author{
Yuki Izumida* \\ Department of Complexity Science and Engineering, Graduate School of Frontier Sciences, The University of Tokyo, \\ Kashiwa 277-8561, Japan
}

(Received 4 May 2020; accepted 26 June 2020; published 21 July 2020)

\begin{abstract}
Low-temperature-differential (LTD) Stirling heat engines are able to operate with a small temperature difference between low-temperature heat reservoirs that exist in our daily lives, and thus they are considered to be an important sustainable energy technology. The author recently proposed a nonlinear dynamics model of an LTD kinematic Stirling heat engine to study the rotational mechanism of the engine [Y. Izumida, Europhys. Lett. 121, 50004 (2018)]. This paper presents our study of the nonequilibrium thermodynamics analysis of this engine model, where a load torque against which the engine does work is introduced. We demonstrate that the engine's rotational state is in a quasilinear response regime where the thermodynamic fluxes show a linear dependence on the thermodynamic forces. Significantly, it is found that the response coefficients of the quasilinear relations are symmetric, which is similar to Onsager symmetry in linear irreversible thermodynamics. Based on these relations, we formulate the maximum efficiency of the engine. We also elucidate that the symmetry of the quasilinear response coefficients emerges by reflecting the (anti-)reciprocity of the Onsager kinetic coefficients identified for the relaxation dynamics of the engine in the vicinity of an equilibrium state. We expect that the present study will pave the way for developing nonequilibrium thermodynamics of autonomous heat engines described as a nonlinear dynamical system.
\end{abstract}

DOI: 10.1103/PhysRevE.102.012142

\section{INTRODUCTION}

The development of heat engines that operate with small temperature differences and at low friction is an important task in heat engine technology. This task has been undertaken by low-temperature-differential (LTD) Stirling heat engines [1-3]. These heat engines were invented by Kolin in the 1980s and subsequently developed primarily by Kolin and Senft [3]. An LTD Stirling heat engine can operate with a small temperature difference between low-temperature heat reservoirs that are available in everyday life, e.g., between the warmth of one's hand and the coldness of the air temperature. Thus, it is considered to be an important sustainable energy technology.

Appropriate mathematical modeling plays an important role in describing and understanding the dynamics of LTD Stirling engines $[4,5]$. The author recently proposed a nonlinear dynamics model of an LTD kinematic Stirling engine to elucidate the rotational mechanism of the engine [6]. In this model, the engine was described as a driven nonlinear pendulum powered by the temperature difference, which obeys simple dynamical equations with only a few

\footnotetext{
*izumida@k.u-tokyo.ac.jp
}

Published by the American Physical Society under the terms of the Creative Commons Attribution 4.0 International license. Further distribution of this work must maintain attribution to the author(s) and the published article's title, journal citation, and DOI. dynamical degrees of freedom. The rotational motion of the engine was described as a stable limit cycle of the dynamical equations sustained by the temperature difference. Moreover, it was shown that the limit cycle disappears via a homoclinic bifurcation [7], with the temperature difference being the bifurcation parameter. The model was recently used to explain the experimental results on an LTD kinematic Stirling engine [8]. It was demonstrated that the core dynamics of the engine are captured by the simple dynamical equations with some modifications that are associated with a few fitting parameters.

The thermodynamic performance analysis of the LTD Stirling heat engines is also an important subject. Although the study in [6] elucidated the rotational mechanism of the engine based on nonlinear dynamics, the thermodynamic performance of the LTD Stirling engine, such as its thermodynamic efficiency, has not yet been formulated. In particular, apart from the present performance of the LTD Stirling engine, it is of interest to formulate its maximum thermodynamic efficiency based on the minimal model.

For small temperature differences, the thermodynamic theories for linear irreversible heat engine have been proposed [9-17], which constitute a branch of finite-time thermodynamics [18-20]. These theories are, however, based on Onsager relations in linear irreversible thermodynamics [21,22], where the linear relations between thermodynamic fluxes and forces can be understood as a perturbation expansion from an equilibrium state. It is not obvious whether such a framework can be applied to the LTD Stirling engine, the rotational motion of which occurs via a nonlinear bifurcation mechanism. Consequently, we need to develop a nonequilibrium 
thermodynamic theory of the LTD Stirling engine described as a nonlinear dynamical system.

In this paper, we develop the nonequilibrium thermodynamics of the LTD kinematic Stirling engine model that was previously introduced [6]. In particular, our goal is to find relevant thermodynamic relations that describe the rotational state of this thermodynamic nonlinear pendulum model, which may be compared to Onsager relations used in describing linear irreversible heat engines. We formulate the thermodynamic efficiency of the LTD kinematic Stirling engine model based on these relations.

The remainder of this paper is organized as follows. In Sec. II, we introduce the LTD kinematic Stirling engine model [6]. In Secs. III and IV, we investigate stationary and rotational states of the engine, respectively, based on the dynamical equations. The formal analytical expressions of the thermodynamic fluxes (angular velocity and heat flux) are derived for the rotational state. In particular, the quasilinear response regime is identified for the rotational state where the thermodynamic fluxes and forces show linear dependency (quasilinear relations), though this regime is not connected to an equilibrium state. In Sec. V, we formulate the thermodynamic efficiency of the engine using the quasilinear relations for which the coefficients turn out to be symmetric. In Sec. VI, we elucidate the origin of the symmetric coefficients in terms of (anti-)reciprocity of the Onsager kinetic coefficients inherited in the relaxation dynamics of the engine. We summarize the study in Sec. VII.

\section{MODEL}

\section{A. Setup}

We use the same model as in our previous study [6], but with a slight extension to add a load torque, which enables the thermodynamic efficiency to be studied. Because the model was previously explained in detail [6], we introduce it here in a simplified but self-contained manner.

The LTD kinematic Stirling engine, regarded as a $\gamma$-type Stirling engine [3], utilizes two connected cylinders (one large and one small) with two movable pistons of different types in these cylinders [Fig. 1(a)]. The working substance of the engine is a gas that is confined to the cylinders. Heat reservoirs at temperatures $T_{\mathrm{b}}$ and $T_{\mathrm{t}}$, such as a warm palm and the cold air surrounding it, are attached to the bottom and top surfaces of the large cylinder, respectively, where we define the temperature difference $\Delta T \equiv T_{\mathrm{b}}-T_{\mathrm{t}}$ and averaged temperature $T_{\mathrm{eq}} \equiv \frac{T_{\mathrm{b}}+T_{\mathrm{t}}}{2}$ for later use. The piston that reciprocates in the large cylinder is a displacer. The motion of the displacer serves to transfer the gas into one side of the cylinders through a small gap between the displacer and the wall of the large cylinder, such that the gas comes into contact with the top and bottom heat reservoirs alternately. In contrast, the small cylinder is fitted with a power piston at the top, and its reciprocating motion constitutes a motive part of the engine. Each piston is connected to a crank with a radius $r$ through a connecting rod, and the reciprocating motion of the power piston is converted into rotational motion via the crank (piston-crank mechanism). The phase angle of the crank connected to the power piston is $\theta(\bmod 2 \pi)$, whereas that of
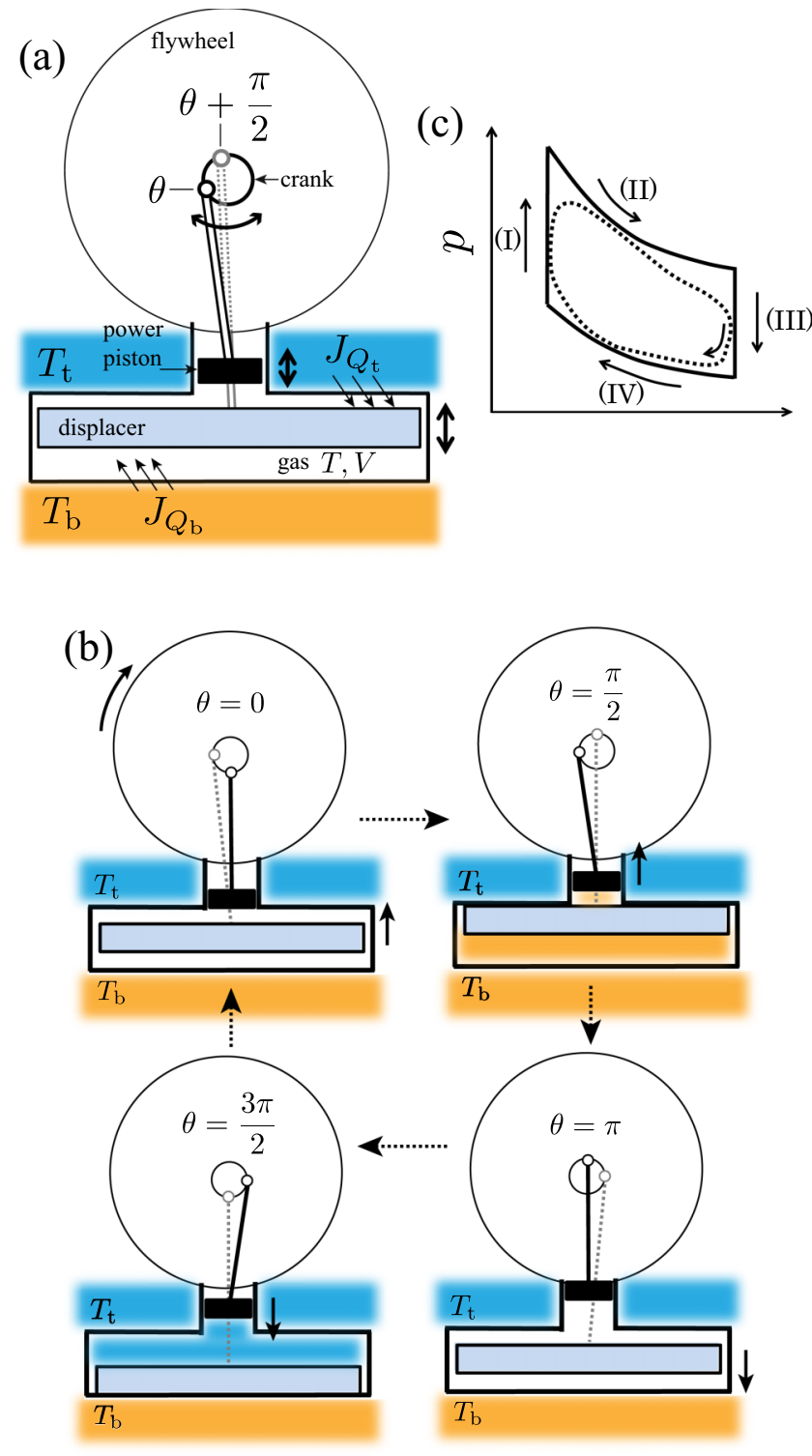

FIG. 1. (a) Schematic of the LTD kinematic Stirling engine. The reciprocating motion of the power piston is converted into the rotational motion of the crank via a piston-crank mechanism. The displacer that advances $\frac{\pi}{2}$ in phase serves to transfer the gas into one side of the cylinder and makes the gas in contact with the bottom and top heat reservoirs. The motive force of the rotation is the temperature difference between the bottom and the top heat reservoirs with temperature $T_{\mathrm{b}}$ and $T_{\mathrm{t}}$, respectively. (b) Schematic of the LTD kinematic Stirling engine steadily rotating clockwise for $\Delta T>0$. (c) Pressure-volume diagram of an ideal Stirling cycle (solid outer cycle) consisting of the four thermodynamic processes (see the text) and the kinematic Stirling engine (dotted inner cycle).

the crank connected to the displacer is fixed as $\theta+\frac{\pi}{2}$ so that it advances in $\frac{\pi}{2}$. The phase angle $\theta$ increases as it rotates clockwise and $\theta=0$ at the lowest height of the power piston [Fig. 1(b)]. The cranks are attached to a flywheel with a large moment of inertia $I$ to smoothen the rotation; the engine can continue to maintain rotation by overcoming $\theta=0$, known as top dead center (TDC), and $\theta=\pi$, known as bottom dead 
center (BDC), at which the reciprocating motion of the piston is not transmitted to the crank.

The phase angle $\theta$ is one of the dynamical variables that expresses the mechanical degree of freedom of this engine model. The other dynamical variable, as a thermodynamic degree of freedom, is the temperature $T$ of the gas. We assume an ideal gas with $f$ internal degrees of freedom as the working substance, for which the equation of state $p V=n R T$ holds. Here, $p$ and $V$ are the pressure and volume of the gas, respectively, and $n$ and $R$ are the amount of substance and gas constant, respectively. The volume $V$ is calculated as the sum of the volume of the large cylinder excluding the volume of the displacer (the swept volume of the displacer during half-stroke), $V_{\mathrm{d}}$, and that of the small cylinder, $V_{\mathrm{p}}(\theta)$ :

$$
V(\theta)=V_{\mathrm{d}}+V_{\mathrm{p}}(\theta)=2 r \sigma_{\mathrm{d}}+s(\theta) \sigma_{\mathrm{p}},
$$

where $\sigma_{\mathrm{d}}$ and $\sigma_{\mathrm{p}}$ are the surface areas of the large and small cylinders, respectively, and

$$
s(\theta) \equiv r(1-\cos \theta)
$$

is the height of the power piston measured from the lowest position corresponding to $\theta=0$.

An ideal Stirling engine cycle repeats an (I) isochoric heating process, (II) isothermal expansion process, (III) isochoric cooling process, and (IV) isothermal compression process [1,2], whose pressure-volume diagram is shown in Fig. 1(c). Conversely, the pressure-volume diagram of an LTD Stirling engine is presented as a circular shape as shown in Fig. 1(c), which is observed in the experiments on LTD kinematic Stirling engines $[8,23]$. While the above thermodynamic processes of the ideal cycle become vaguer and may not be fully discriminated from each other for an LTD Stirling engine, they can operate autonomously without being controlled by external agents. Therefore, in Sec. II B, we introduce the dynamical equations of our engine model [6].

\section{B. Dynamical equations}

The set of equations that describe our LTD kinematic Stirling engine constitute the equation of motion of the power piston, equation of motion of the crank, and time-evolution equation of the gas temperature given as the energy conservation law (the first law of thermodynamics):

$$
\begin{gathered}
m_{\mathrm{p}} \frac{d^{2} s}{d t^{2}}=\sigma_{\mathrm{p}}\left(\frac{n R T}{V(\theta)}-p_{\text {air }}-\frac{F_{\text {int }}}{\sigma_{\mathrm{p}}}\right)-\Gamma_{\mathrm{p}} \frac{d s}{d t}, \\
I \frac{d^{2} \theta}{d t^{2}}=r F_{\text {int }} \sin \theta-\Gamma \frac{d \theta}{d t}-T_{\text {load }}, \\
\frac{f}{2} n R \frac{d T}{d t}=\sum_{m=\mathrm{b}, \mathrm{t}} J_{Q_{m}}-\left(p_{\text {air }}+\frac{F_{\text {int }}}{\sigma_{\mathrm{p}}}\right) \frac{d V}{d t} .
\end{gathered}
$$

Here, $m_{\mathrm{p}}$ and $\Gamma_{\mathrm{p}}$ in Eq. (3) are the mass of the power piston and friction coefficient associated with the power piston, respectively. Further, $F_{\text {int }}$ in Eqs. (3)-(5) is the action-reaction force between the power piston and the crank $[5,24] . \Gamma$ and $T_{\text {load }}$ in Eq. (4) are the friction coefficient associated with the crank and load torque acting on the crank, respectively. $p_{\text {air }}$ in Eqs. (3) and (5) is the atmospheric pressure acting on the power piston. The rate of internal energy change of the gas on the left-hand side of Eq. (5) is equated to the heat fluxes and work flux on the right-hand side. The heat fluxes from the bottom and top surfaces of the large cylinder obey the Fourier law [Fig. 1(a)]:

$$
J_{Q_{m}}=G_{m}(\theta)\left(T_{m}-T\right) .
$$

$G_{m}(\theta)(m=\mathrm{b}, \mathrm{t})$ is defined as [6]

$$
G_{m}(\theta) \equiv G \chi_{m}(\theta),
$$

where $G$ is the thermal conductance associated with the heat transfer between the gas and the surface of the large cylinder, and $\chi_{m}(\theta)\left(0 \leqslant \chi_{m}(\theta) \leqslant 1\right)$, defined as

$$
\chi_{\mathrm{b}}(\theta) \equiv \frac{1+\sin \theta}{2}, \quad \chi_{\mathrm{t}}(\theta) \equiv 1-\chi_{\mathrm{b}}(\theta)=\frac{1-\sin \theta}{2},
$$

is a function that controls the coupling between the gas and the bottom or top heat reservoir depending on the phase angle [6]. The role of the displacer transferring the gas into one side of the cylinders is represented by the function Eq. (8). Then we can revise

$$
\sum_{m=\mathrm{b}, \mathrm{t}} J_{Q_{m}}=G\left(T_{\mathrm{eff}}(\theta)-T\right),
$$

where $T_{\text {eff }}(\theta)$ is the effective temperature that periodically changes, depending on the phase angle $\theta$, as

$$
\begin{gathered}
T_{\text {eff }}(\theta) \equiv T_{\mathrm{t}}+\chi_{\mathrm{b}}(\theta) \Delta T=T_{\mathrm{t}}+\frac{1+\sin \theta}{2} \Delta T \\
=T_{\text {eq }}+\frac{\sin \theta}{2} \Delta T .
\end{gathered}
$$

We can thus consider the gas as though it were in contact with a single heat reservoir, the temperature of which dynamically oscillates in a sinusoidal manner between $T_{\mathrm{b}}$ at $\theta=\frac{\pi}{2}$ $\left[\chi_{\mathrm{b}}\left(\frac{\pi}{2}\right)=1\right.$ and $\left.\chi_{\mathrm{t}}\left(\frac{\pi}{2}\right)=0\right]$ and $T_{\mathrm{t}}$ at $\theta=\frac{3 \pi}{2}\left[\chi_{\mathrm{b}}\left(\frac{3 \pi}{2}\right)=0\right.$ and $\left.\chi_{\mathrm{t}}\left(\frac{3 \pi}{2}\right)=1\right]$, which loosely approximates the ideal Stirling thermodynamic cycle [6].

We assume that the mass of the power piston and friction coefficient in Eq. (3) are negligible, as $m_{\mathrm{p}}=\Gamma_{\mathrm{p}}=0$. We then obtain $F_{\text {int }}=\sigma_{\mathrm{p}}\left(\frac{n R T}{V(\theta)}-p_{\text {air }}\right)$ from Eq. (3). By inserting this into Eqs. (4) and (5), and noting Eq. (9), we obtain

$$
\begin{gathered}
I \frac{d^{2} \theta}{d t^{2}}=r \sigma_{\mathrm{p}}\left(\frac{n R T}{V(\theta)}-p_{\mathrm{air}}\right) \sin \theta-\Gamma \frac{d \theta}{d t}-T_{\mathrm{load}}, \\
\frac{f}{2} n R \frac{d T}{d t}=G\left(T_{\mathrm{eff}}(\theta)-T\right)-\frac{n R T}{V(\theta)} \frac{d V}{d t} .
\end{gathered}
$$

Subsequently, Eqs. (12) and (13) are expressed in terms of the three-dimensional dynamical system as

$$
\begin{gathered}
\frac{d \theta}{d t}=\omega, \\
\frac{d \omega}{d t}=\frac{\sigma_{\mathrm{p}}}{I}\left(\frac{n R T}{V(\theta)}-p_{\text {air }}\right) r \sin \theta-\frac{\Gamma}{I} \omega-\frac{T_{\text {load }}}{I}, \\
\frac{d T}{d t}=\frac{2 G}{f n R}\left(T_{\text {eff }}(\theta)-T\right)-\frac{2 \operatorname{Tr} \sigma_{\mathrm{p}} \sin \theta}{f V(\theta)} \omega,
\end{gathered}
$$

where $\omega$ denotes the angular velocity. By assuming a timescale separation between the crank and the gas dynamics, we can make the adiabatic approximation $\frac{d T}{d t}=0$ by regarding $T$ as a fast variable and $\theta$ and $\omega$ as slow variables [25]. By 
formally substituting $\frac{d T}{d t}=0$ into Eq. (16) and solving it with respect to $T$, we have the adiabatic approximation solution

$$
T(\theta, \omega)=\frac{T_{\mathrm{eff}}(\theta)}{1+\frac{n R r \sigma_{\mathrm{p}} \sin \theta}{G V(\theta)} \omega},
$$

which is determined by the slow variables $\theta$ and $\omega$ of the crank (see Appendix A for the detailed derivation). This approximation indicates that the motion of the piston and crank is considered as an externally controlled parameter for the gas, rather than being dynamically determined by the coupled equations in Eqs. (12) and (13) involving the gas dynamics. By substituting Eq. (17) into Eq. (15), we adiabatically eliminate $T$ and obtain the following two-dimensional dynamical system:

$$
\begin{gathered}
\frac{d \theta}{d t}=\omega, \\
\frac{d \omega}{d t}=\frac{\sigma_{\mathrm{p}}}{I}\left(\frac{n R T(\theta, \omega)}{V(\theta)}-p_{\mathrm{air}}\right) r \sin \theta-\frac{\Gamma}{I} \omega-\frac{T_{\mathrm{load}}}{I} .
\end{gathered}
$$

These dynamical equations describe the engine as a nonlinear pendulum driven by the temperature difference. In particular, as we see in Sec. IVC, the term that is proportional to $\sin ^{2} \theta \Delta T$ constituted with $\sin \theta \Delta T$ in $T_{\text {eff }}(\theta)$ and $\sin \theta$ for the rotational torque represents an effective driving force for the steadily rotating engine, which does not vanish upon cycle-averaging. Equations (18) and (19) [or Eqs. (14)-(16) before the adiabatic approximation] are the basic dynamical equations of our LTD kinematic Stirling engine model. The stationary and rotational states of the engine are described as a fixed point and stable limit cycle of Eqs. (18) and (19), respectively, which coexist depending on the parameters [6].

For numerical calculations, we use nondimensionalized equations [6]. In the text, we use the two-dimensional dynamical model, Eqs. (18) and (19), whose nondimensionalized equations become

$$
\begin{gathered}
\frac{d \theta}{d \tilde{t}}=\tilde{\omega}, \\
\frac{d \tilde{\omega}}{d \tilde{t}}=\tilde{\sigma}\left(\frac{\tilde{T}(\theta, \tilde{\omega})}{\tilde{V}(\theta)}-\tilde{p}_{\text {air }}\right) \sin \theta-\tilde{\Gamma} \tilde{\omega}-\tilde{T}_{\mathrm{load}},
\end{gathered}
$$

where

$$
\tilde{T}(\theta, \tilde{\omega})=\frac{\tilde{T}_{\mathrm{eff}}(\theta)}{1+\frac{\tilde{\sigma} \sin \theta \tilde{\omega}}{\tilde{G} \tilde{V}(\theta)}} .
$$

Here, the following nondimensionalized quantities are used: $\tilde{t}=\sqrt{\frac{n R T_{\mathrm{eq}}}{I}} t, \tilde{\omega}=\frac{\omega}{\sqrt{\frac{n R T_{\mathrm{eq}}}{I}}}, \tilde{G}=\frac{G}{n R \sqrt{\frac{n R T_{\mathrm{eq}}}{I}}}, \tilde{\sigma}=\frac{\sigma_{\mathrm{p}}}{\sigma_{d}}, \tilde{\Gamma}=\frac{\Gamma}{\sqrt{n R T_{\mathrm{eq}}}}$, $\tilde{p}_{\text {air }}=\frac{\sigma_{d} r p_{\text {air }}}{n R T_{\text {eq }}}, \quad \tilde{T}_{\text {load }}=\frac{T_{\text {lad }}}{n R T_{\text {eq }}}, \quad$ and $\Delta \tilde{T}=\frac{\Delta T}{T_{\text {eq }}}$. The quantities with a tilde denote the nondimensionalized quantities throughout the paper. $\tilde{T}_{\text {eff }}(\theta)=1+\frac{\sin \theta}{2} \Delta \tilde{T}$ and $\tilde{V}(\theta)=2+$ $\tilde{\sigma}(1-\cos \theta)$ are the nondimensionalized effective temperature and volume, respectively. In the text, we use $\tilde{\sigma}=0.02$, $\tilde{p}_{\text {air }}=\frac{1}{\tilde{V}\left(\frac{\pi}{4}\right)}=\frac{1}{2+\tilde{\sigma}\left(1-\cos \left(\frac{\pi}{4}\right)\right)} \simeq 0.49854, \tilde{G}=1.5$, and $\tilde{\Gamma}=$ 0.001 and vary $\Delta \tilde{T}$ and $\tilde{T}_{\text {load }}$ to investigate the engine's working regime. Under these parameters, the adiabatic elimination serves as a good approximation and the friction coefficient is sufficiently small for the engine to be able to operate in a low-temperature differential. In Appendix B, we also use the nondimensionalized Eqs. (14)-(16) for comparing the twodimensional and the three-dimensional dynamical models. For numerical calculations, we use the fourth-order Runge-Kutta method with time step $\Delta t=0.01$.

\section{STATIONARY STATES}

\section{A. Thermodynamic branches and dead-center branches}

We investigate the fixed points $\left(\theta^{*}, \omega^{*}\right)$ of Eqs. (18) and (19) satisfying $\frac{d \theta}{d t}=\frac{d \omega}{d t}=0$ as the stationary states of the engine. For the equilibrium condition $\Delta T=0$ and $T_{\text {load }}=0$, $\left(\theta_{\mathrm{eq} 1}, 0\right)=\left(\theta_{\mathrm{eq}}, 0\right)$, and $\left(\theta_{\mathrm{eq} 2}, 0\right)=\left(2 \pi-\theta_{\mathrm{eq}}, 0\right)$ are the fixed points of Eqs. (18) and (19), where $\theta_{\mathrm{eq}}$ satisfies the pressure equilibrium condition $\frac{n R T_{\mathrm{eq}}}{V\left(\theta_{\mathrm{eq}}\right)}-p_{\text {air }}=0$ and thus expresses an equilibrium state. Because of the symmetry $V\left(\theta_{\mathrm{eq}}\right)=V(2 \pi-$ $\left.\theta_{\mathrm{eq}}\right), 2 \pi-\theta_{\mathrm{eq}}$ also satisfies the condition. Depending on the parameters, these fixed points may not exist. We also have $(0,0)$ and $(\pi, 0)$ as the other fixed points of Eqs. (18) and (19) for $\Delta T=0$ and $T_{\text {load }}=0$, which represent the stationary states at the dead centers and exist for any parameter. Thus, there is a maximum of four fixed points of Eqs. (18) and (19).

When the nonvanishing $\Delta T$ and $T_{\text {load }}$ are applied, the fixed points $\left(\theta_{\mathrm{eq} 1}, 0\right)$ and $\left(\theta_{\mathrm{eq} 2}, 0\right)$ corresponding to the equilibrium state change to $\left(\theta_{\mathrm{th} 1}, 0\right)$ and $\left(\theta_{\mathrm{eq} 2}, 0\right)$, where $\theta_{\mathrm{th} 1}$ and $\theta_{\mathrm{th} 2}$ constitute thermodynamic branches. The fixed points $(0,0)$ and $(\pi, 0)$ also change to $\left(\theta_{\mathrm{TDC}}, 0\right)$ and $\left(\theta_{\mathrm{BDC}}, 0\right)$, where $\theta_{\mathrm{TDC}}$ and $\theta_{\mathrm{BDC}}$ constitute dead-center branches. Here, $\theta_{\mathrm{th} 1}, \theta_{\mathrm{th} 2}$, $\theta_{\mathrm{TDC}}$, and $\theta_{\mathrm{BDC}}$ are given as the solution $\theta^{*}$ of the following equation as the condition of fixed points as

$$
0=\sigma_{\mathrm{p}}\left(\frac{n R T_{\text {eff }}\left(\theta^{*}\right)}{V\left(\theta^{*}\right)}-p_{\text {air }}\right) r \sin \theta^{*}-T_{\text {load }},
$$

where $\left.\theta^{*}\right|_{\left(\Delta T, T_{\text {load }}\right)=(0,0)}=\theta_{\text {eq1 }}$ and $\left.\theta^{*}\right|_{\left(\Delta T, T_{\text {load }}=(0,0)\right.}=\theta_{\text {eq2 } 2}$ for the thermodynamic branches $\theta_{\text {th1 }}$ and $\theta_{\text {th2 }}$, respectively, and $\left.\theta^{*}\right|_{\left(\Delta T, T_{\text {load }}\right)=(0,0)}=0$ and $\theta_{\left(\Delta T, T_{\text {load }}\right)=(0,0)}^{*}=\pi$ for the deadcenter branches $\theta_{\mathrm{TDC}}$ and $\theta_{\mathrm{BDC}}$, respectively.

The stability of the fixed points on the thermodynamic and dead-center branches is investigated by checking the determinant $\Delta$ and trace $\mathcal{T}$, calculated from the linearized equations of Eqs. (18) and (19) as [7]

$$
\begin{gathered}
\Delta=-\frac{\sigma_{\mathrm{p}}}{I} \frac{n R r \cos \theta^{*} \sin \theta^{*}}{2 V\left(\theta^{*}\right)} \Delta T+\frac{\sigma_{\mathrm{p}}^{2}}{I} \frac{n R r^{2} T_{\mathrm{eff}}\left(\theta^{*}\right) \sin ^{2} \theta^{*}}{V^{2}\left(\theta^{*}\right)} \\
-\frac{\sigma_{\mathrm{p}}}{I}\left(\frac{n R T_{\mathrm{eff}}\left(\theta^{*}\right)}{V\left(\theta^{*}\right)}-p_{\text {air }}\right) r \cos \theta^{*} \\
\mathcal{T}=-\frac{\sigma_{\mathrm{p}}^{2}}{I} \frac{n^{2} R^{2} r^{2} T_{\mathrm{eff}}\left(\theta^{*}\right) \sin ^{2} \theta^{*}}{G V^{2}\left(\theta^{*}\right)}-\frac{\Gamma}{I}
\end{gathered}
$$

respectively, where $\theta^{*}$ is given as the solution of Eq. (23). Figure 2(a) shows the four branches for $T_{\text {load }}=0$, where the solid and dashed curves denote the stable fixed point and unstable fixed point (saddle point), respectively. For the given parameters, we have $\theta_{\mathrm{eq} 1}=\frac{\pi}{4}$ and $\theta_{\mathrm{eq} 2}=\frac{7 \pi}{4}$, and we thus have the thermodynamic branches $\theta_{\text {th1 }}$ and $\theta_{\text {th2 }}$ satisfying $\left.\theta_{\text {th } 1}\right|_{\left(\Delta T, T_{\text {load }}\right)=(0,0)}=\frac{\pi}{4}$ and $\left.\theta_{\text {th } 2}\right|_{\left(\Delta T, T_{\text {load }}\right)=(0,0)}=\frac{7 \pi}{4}$.

In the vicinity of the equilibrium state $\theta_{\text {eq }}$, the thermodynamic branch $\theta_{\text {th }}$ can be expanded as $\theta_{\text {th }} \simeq \theta_{\text {eq }}+$ $a_{1} \tilde{T}_{\text {load }}+a_{2} \Delta \tilde{T}$, where $a_{i}$ are the expansion coefficients to be 
(a)
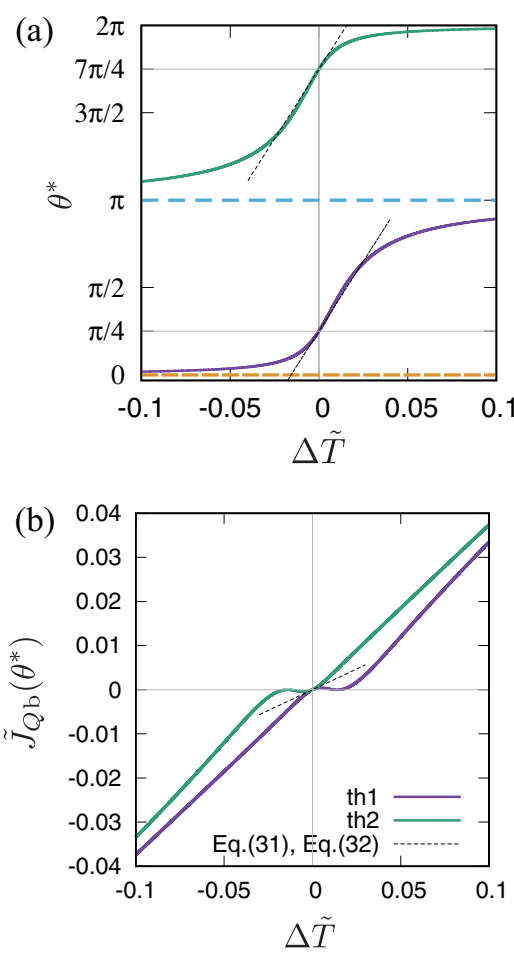

FIG. 2. (a) Thermodynamic and dead-center branches for $T_{\text {load }}=$ 0 with the linear response lines given by Eqs. (27) and (28). Solid and dashed curves represent the stable fixed point $(\mathcal{T}>0$ and $\Delta>0)$ and saddle point $(\Delta<0)$, respectively [see Eqs. (24) and (25)]. (b) (Nondimensionalized) heat fluxes on the stable thermodynamic branches for $T_{\text {load }}=0$ with the linear response lines given by Eqs. (31) and (32).

determined. By substituting this expansion into Eq. (23), we obtain

$$
\theta_{\mathrm{th}} \simeq \theta_{\mathrm{eq}}-\frac{\tilde{V}^{2}\left(\theta_{\mathrm{eq}}\right)}{\tilde{\sigma}^{2} \sin ^{2} \theta_{\mathrm{eq}}} \tilde{T}_{\mathrm{load}}+\frac{\tilde{V}\left(\theta_{\mathrm{eq}}\right)}{2 \tilde{\sigma}} \Delta \tilde{T}
$$

For the present case of $\theta_{\text {eq } 1}=\frac{\pi}{4}$ and $\theta_{\text {eq } 2}=\frac{7 \pi}{4}$, we can easily obtain

$\theta_{\mathrm{th} 1} \simeq \frac{\pi}{4}-\frac{2\left(2+\tilde{\sigma}\left(1-\frac{1}{\sqrt{2}}\right)\right)^{2}}{\tilde{\sigma}^{2}} \tilde{T}_{\mathrm{load}}+\frac{2+\tilde{\sigma}\left(1-\frac{1}{\sqrt{2}}\right)}{2 \tilde{\sigma}} \Delta \tilde{T}$,

$\theta_{\mathrm{th} 2} \simeq \frac{7 \pi}{4}-\frac{2\left(2+\tilde{\sigma}\left(1-\frac{1}{\sqrt{2}}\right)\right)^{2}}{\tilde{\sigma}^{2}} \tilde{T}_{\mathrm{load}}+\frac{2+\tilde{\sigma}\left(1-\frac{1}{\sqrt{2}}\right)}{2 \tilde{\sigma}} \Delta \tilde{T}$.

The linear response lines of $\theta_{\text {th }}$ from the original equilibrium value $\theta_{\text {eq }}$ are shown in Fig. 2(a).

\section{B. Heat fluxes at stationary states}

For the nonvanishing $\Delta T$, the engine conducts heat from the hot heat reservoir to the cold heat reservoir at the stationary states. The heat flux from each heat reservoir into the gas at the stationary state is given by

$$
\begin{aligned}
& J_{Q_{\mathrm{b}}}\left(\theta^{*}\right)=G_{\mathrm{b}}\left(\theta^{*}\right)\left(T_{\mathrm{b}}-T\left(\theta^{*}, 0\right)\right)=G \frac{\cos ^{2} \theta^{*}}{4_{2}} \Delta T, \\
& J_{Q_{\mathrm{t}}}\left(\theta^{*}\right)=G_{\mathrm{t}}\left(\theta^{*}\right)\left(T_{\mathrm{t}}-T\left(\theta^{*}, 0\right)\right)=-G \frac{\cos ^{*} \theta^{4}}{4} \Delta T,
\end{aligned}
$$

with $\frac{G}{4} \cos ^{2} \theta^{*}$ being an effective thermal conductance that depends on $\theta^{*}$. Figure 2(b) shows the (nondimensionalized) heat fluxes $\tilde{J}_{Q_{\mathrm{b}}}\left(\theta^{*}\right)=\frac{J_{Q_{\mathrm{b}}}\left(\theta^{*}\right)}{n R T_{\text {eq }} \sqrt{\frac{n R T_{\mathrm{eq}}}{I}}}$ on the stable thermodynamic branches $\theta^{*}=\theta_{\mathrm{th} 1}, \theta_{\mathrm{th} 2}$ corresponding to those in Fig. 2(a), where we can approximate $J_{Q_{\mathrm{b}}}\left(\theta_{\mathrm{th} 1}\right)$ and $J_{Q_{\mathrm{b}}}\left(\theta_{\mathrm{th} 2}\right)$ as

$$
\begin{aligned}
& J_{Q_{\mathrm{b}}}\left(\theta_{\mathrm{th} 1}\right) \simeq G \frac{\cos ^{2}\left(\frac{\pi}{4}\right)}{4} \Delta T=\frac{G}{8} \Delta T, \\
& J_{Q_{\mathrm{b}}}\left(\theta_{\mathrm{th} 2}\right) \simeq G \frac{\cos ^{2}\left(\frac{7 \pi}{4}\right)}{4} \Delta T=\frac{G}{8} \Delta T
\end{aligned}
$$

in the vicinity of the equilibrium state, by using

$$
J_{Q_{\mathrm{b}}}\left(\theta_{\mathrm{th}}\right) \simeq G \frac{\cos ^{2} \theta_{\mathrm{eq}}}{4} \Delta T
$$

in Eq. (29).

\section{ROTATIONAL STATE}

\section{A. Numerical calculations of time-averaged angular velocity and heat fluxes}

We investigate the stable limit cycle of Eqs. (18) and (19) representing the rotational state of the engine. Denoting one cycle period of the stable limit cycle by $\tau$, we define the timeaveraged angular velocity and heat fluxes as

$$
\begin{gathered}
\langle\omega\rangle \equiv \frac{1}{\tau} \int_{0}^{\tau} \omega d t=\frac{1}{\tau} \int_{0}^{\tau} \frac{d \theta}{d t} d t=\frac{2 \pi}{\tau} \\
\left\langle J_{Q_{m}}\right\rangle \equiv \frac{1}{\tau} \int_{0}^{\tau} J_{Q_{m}} d t=\frac{1}{\tau} \int_{0}^{\tau} G_{m}(\theta)\left(T_{m}-T(\theta, \omega)\right) d t
\end{gathered}
$$

respectively, where $\langle\cdots\rangle \equiv \frac{1}{\tau} \int_{0}^{\tau} \ldots d t$ denotes a time average and $T(\theta, \omega)$ in Eq. (35) is given by Eq. (17).

In Fig. 3(a), we present the $\langle\tilde{\omega}\rangle-\tilde{T}_{\text {load }}$ curve of the stable limit cycle. See also Fig. 3(b) for the corresponding thermodynamic and dead-center branches.

For sufficiently small $\tilde{T}_{\text {load }}>0$, the engine is able to rotate against the load torque, producing positive work $(\langle\tilde{\omega}\rangle>0)$. As $\tilde{T}_{\text {load }}$ increases, the engine stops rotating at $\tilde{T}_{\text {load }}^{\prime} \simeq$ $7.0125 \times 10^{-5}$, which is the bifurcation point of the stable limit cycle. As $\tilde{T}_{\text {load }}$ increases further and exceeds the bifurcation point $T_{\text {load }}^{\prime \prime} \simeq 9.9027 \times 10^{-5}$, the stable limit cycle appears again; the engine is able to rotate again, but in the same direction as the applied load torque $(\langle\tilde{\omega}\rangle<0)$. $\langle\tilde{\omega}\rangle$ shows the linear dependency on $\tilde{T}_{\text {load }}$ as it deviates sufficiently from the bifurcation points. This linear dependency for the two-dimensional dynamical model, Eqs. (18) and (19), will be theoretically confirmed in Sec. IV C. We note that such linear dependency is not observed for the three-dimensional dynamical model, Eqs. (14)-(16), with parameter ranges for which the adiabatic approximation is not valid (Appendix B).

The above bifurcations are homoclinic bifurcations [7]. To illustrate this for the bifurcation at $\tilde{T}_{\text {load }}^{\prime}$, we show the orbit of the stable limit cycle on the phase plane in Fig. 4(a) 

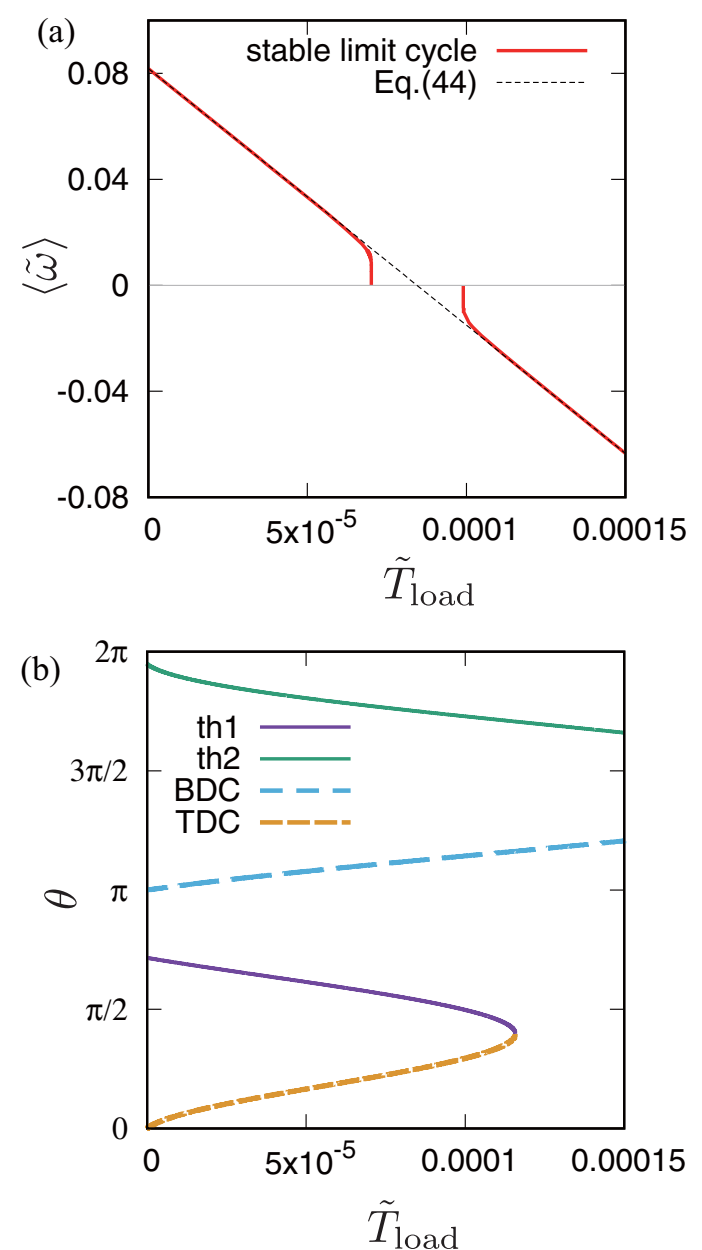

FIG. 3. (a) $\langle\tilde{\omega}\rangle-\tilde{T}_{\text {load }}$ curve of the stable limit cycle for $\Delta \tilde{T}=$ 1/29.3. The dashed line denotes the theoretical line given in Eq. (44). (b) Thermodynamic branches and dead-center branches as a function of the load torque for $\Delta \tilde{T}=1 / 29.3$. Solid and dashed curves represent the stable fixed point $(\mathcal{T}>0$ and $\Delta>0)$ and saddle point $(\Delta<0)$, respectively [see Eqs. (24) and (25)]. There are one or two stable fixed points, depending on the value of $\tilde{T}_{\text {load }}$.

and the period $\tilde{\tau}=\frac{2 \pi}{\langle\tilde{\omega}\rangle}$ in Fig. 4(b) in the vicinity of $\tilde{T}_{\text {load }}^{\prime}$. In Fig. 4(a), we can see that the orbit of the stable limit cycle closely passes the saddle point on the BDC branch in Fig. 3(b) by taking a long time. At the bifurcation point, part of the orbit touches the saddle point and the stable limit cycle disappears, forming a homoclinic orbit [7]. Thus, although the dead-center branch is not connected to the equilibrium state, the saddle point on the branch plays an important role in the homoclinic bifurcation of the limit cycle. As characteristics of the homoclinic bifurcation, the period of the limit cycle exhibits slow divergence according to the theoretical prediction $\tilde{\tau} \propto-\log \left(\tilde{T}_{\text {load }}^{\prime}-\tilde{T}_{\text {load }}\right)$ [7], which is confirmed in Fig. 4(b). This slow divergence indicates a steep change in the angular velocity $\langle\tilde{\omega}\rangle=\frac{2 \pi}{\tilde{\tau}}$ near the bifurcation points, as shown in Fig. 3(a).

We show the $\tilde{T}_{\text {load }}$ dependence of $\left\langle\tilde{J}_{Q_{\mathrm{b}}}\right\rangle$ in Fig. 5(a). $\left\langle\tilde{J}_{Q_{\mathrm{b}}}\right\rangle$ shows the linear dependency on $\tilde{T}_{\text {load }}$ as it deviates sufficiently from the bifurcation points in the same manner as $\langle\tilde{\omega}\rangle$ in Fig. 3(a). This linear dependency is further investigated in
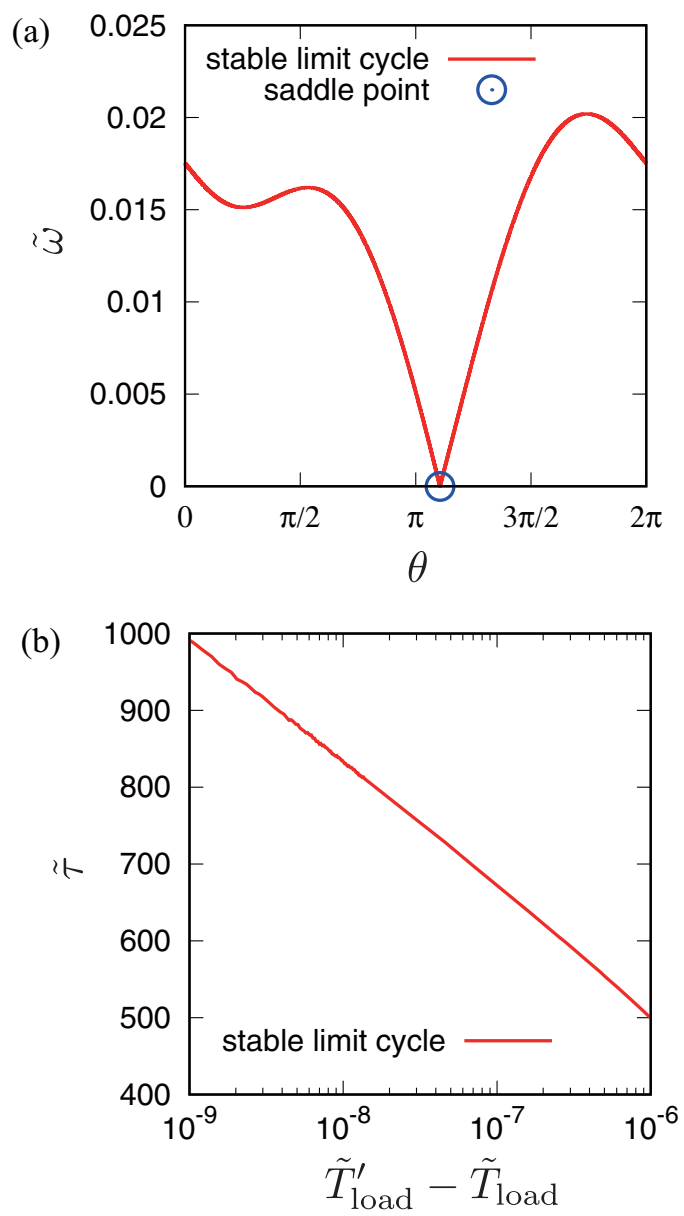

FIG. 4. (a) Orbit of the stable limit cycle on the phase plane near the bifurcation point $\tilde{T}_{\text {load }} \simeq \tilde{T}_{\text {load }}^{\prime}$ for $\Delta \tilde{T}=1 / 29.3$. The saddle point is located on the BDC branch in Fig. 3(b). (b) Semilog plot of the period $\tilde{\tau}$ as a function of $\tilde{T}_{\text {load }}^{\prime}-\tilde{T}_{\text {load }}$ near the bifurcation point $\tilde{T}_{\text {load }}^{\prime}$.

Sec. IV D. As $\tilde{T}_{\text {load }}$ approaches the bifurcation points, we find that $\left\langle\tilde{J}_{Q_{\mathrm{b}}}\right\rangle$ deviates from the linear line and slowly converges to a constant value. This behavior is associated with the homoclinic bifurcation, which is clarified in Sec. IV C.

\section{B. Derivation of formal analytical expressions}

We derive formal analytical expressions of the timeaveraged fluxes $\langle\omega\rangle$ and $\left\langle J_{Q_{\mathrm{b}}}\right\rangle$ for a small temperature difference and load torque, to explain their behaviors as we have seen in Sec. IV A. We first derive a formal analytical expression of $\langle\omega\rangle$ using Eqs. (18) and (19). We assume that $(\theta, \omega)$ is the stable limit cycle with period $\tau$ of Eqs. (18) and (19). Then, time-averaging both sides of Eq. (19) yields

$$
0=\frac{\sigma_{\mathrm{p}}}{I}\left\langle\left(\frac{n R T(\theta, \omega)}{V(\theta)}-p_{\text {air }}\right) r \sin \theta\right\rangle-\frac{\Gamma}{I}\langle\omega\rangle-\frac{T_{\text {load }}}{I} .
$$

Note that the inertia term on the left-hand side has vanished as $\left\langle\frac{d \omega}{d t}\right\rangle=\frac{1}{\tau} \int_{0}^{\tau} \frac{d \omega}{d t} d t=\frac{1}{\tau}[\omega]_{0}^{\tau}=0$. We can then approximate Eq. (17) as

$$
T(\theta, \omega)=T_{\mathrm{eff}}(\theta)-T_{\mathrm{eq}} \frac{r \sin \theta \sigma_{\mathrm{p}}}{\tilde{G} V(\theta)} \tilde{\omega}+O\left(\Delta \tilde{T} \tilde{\omega}, \tilde{\omega}^{2}\right),
$$



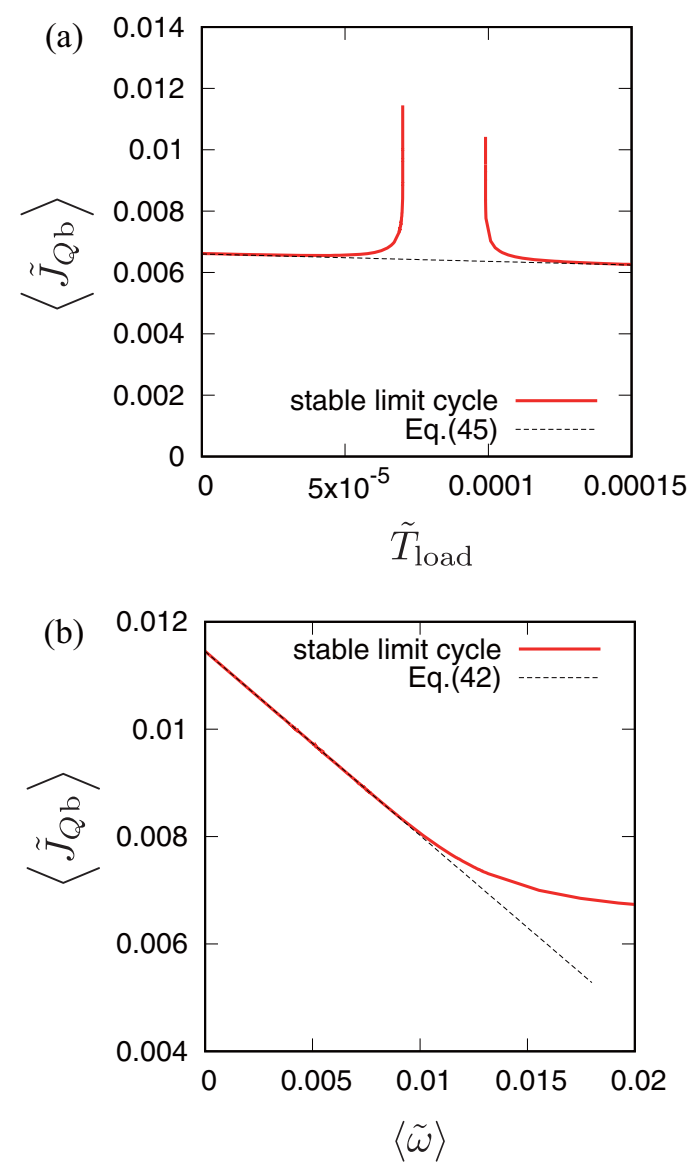

FIG. 5. (a) $\left\langle\tilde{J}_{Q_{\mathrm{b}}}\right\rangle-\tilde{T}_{\text {load }}$ curve of the stable limit cycle for $\Delta \tilde{T}=$ $1 / 29.3$. The dashed line denotes the theoretical line given by Eq. (45). (b) $\left\langle\tilde{J}_{Q_{\mathrm{b}}}\right\rangle$ in the vicinity of the bifurcation point $\tilde{T}_{\text {load }}^{\prime}$ with the theoretical line given in Eq. (42). $\theta_{\mathrm{H}}$ in Eq. (42) is estimated as $\theta_{\mathrm{H}} \simeq$ 3.4722 at $\tilde{T}_{\text {load }} \simeq \tilde{T}_{\text {load }}^{\prime}$. The (nondimensionalized) coefficient $\tilde{a}=$ $\frac{a}{n R T_{\mathrm{eq}}}$ is estimated as $\tilde{a} \simeq-0.35838$ using a least-squares method.

assuming that $|\Delta \tilde{T}|$ and $|\tilde{\omega}|$ are sufficiently small. By using Eq. (37), we can rewrite the first term (rotational torque term) on the right-hand side of Eq. (36) as

$$
\begin{aligned}
& \frac{\sigma_{\mathrm{p}}}{I}\left\langle\left(\frac{n R T(\theta, \omega)}{V(\theta)}-p_{\text {air }}\right) r \sin \theta\right\rangle \\
& \simeq \frac{\sigma_{\mathrm{p}}}{I}\left\langle\left(\frac{n R T_{\mathrm{eff}}(\theta)}{V(\theta)}-\frac{n^{2} R^{2} T_{\mathrm{eq}} r \sin \theta \sigma_{\mathrm{p}}}{G V^{2}(\theta)} \omega-p_{\text {air }}\right) r \sin \theta\right\rangle .
\end{aligned}
$$

From Eqs. (36) and (38), we obtain

$$
\langle\omega\rangle=\frac{\left\langle\sigma_{\mathrm{p}}\left(\frac{n R T_{\mathrm{eff}}(\theta)}{V(\theta)}-p_{\mathrm{air}}\right) r \sin \theta\right\rangle-T_{\mathrm{load}}}{\Gamma+\frac{\sigma_{\mathrm{p}}^{2} n^{2} R^{2} T_{\mathrm{eq}} r^{2}}{G}\left\langle\frac{\sin ^{2} \theta}{V^{2}(\theta)}\right\rangle_{\theta}},
$$

where $\langle\cdots\rangle_{\theta} \equiv \frac{1}{2 \pi} \int_{0}^{2 \pi} \ldots d \theta$ denotes a phase average. This formal analytical expression states that the averaged angular velocity is determined by the time average of the rotational torque and load torque.

We next derive a formal analytical expression of the timeaveraged heat flux $\left\langle J_{Q_{b}}\right\rangle$. Under the approximation of Eq. (37), the heat flux $\left\langle J_{Q_{\mathrm{b}}}\right\rangle$ in Eq. (35) is approximated as

$$
\begin{aligned}
\left\langle J_{Q_{\mathrm{b}}}\right\rangle= & \frac{1}{\tau} \int_{0}^{\tau} G_{\mathrm{b}}(\theta)\left(T_{\mathrm{b}}-T(\theta, \omega)\right) d t \\
\simeq & \frac{1}{\tau} \int_{0}^{\tau} G \frac{1+\sin \theta}{2}\left(T_{\mathrm{b}}-T_{\mathrm{eff}}(\theta)\right. \\
& \left.+T_{\mathrm{eq}} \frac{n R r \sin \theta \sigma_{\mathrm{p}}}{G V(\theta)} \omega\right) d t
\end{aligned}
$$

By using $\tau=\frac{2 \pi}{\langle\omega\rangle}$ and noting that $\omega=\frac{d \theta}{d t}$, we obtain

$$
\left\langle J_{Q_{\mathrm{b}}}\right\rangle=\frac{G}{4}\left\langle\cos ^{2} \theta\right\rangle \Delta T+\frac{T_{\mathrm{eq}} n \operatorname{Rr} \sigma_{\mathrm{p}}}{2}\left\langle\frac{\sin ^{2} \theta}{V(\theta)}\right\rangle_{\theta}\langle\omega\rangle,
$$

where $\langle\omega\rangle$ is given in Eq. (39). The first term on the right-hand side of Eq. (41) is the time-averaged heat flux formally obeying the Fourier law, with $\frac{G}{4}\left\langle\cos ^{2} \theta\right\rangle$ being the time-averaged thermal conductance. However, this is not similar to the heat leakage at the stationary state in Eq. (29) because of its strong correlation with the engine's rotational motion through the time-averaged thermal conductance. The second term on the right-hand side of Eq. (41) represents the heat transfer in proportion to the averaged angular velocity, which is also caused by the engine's rotational motion.

\section{Near the bifurcation point}

Near the bifurcation point, the orbit of the limit cycle stays in proximity to the saddle point almost all the time [Fig. 4(a)]. Thus, the effective thermal conductance $\frac{G}{4}\left\langle\cos ^{2} \theta\right\rangle$ in Eq. (41) is approximated as $\frac{G}{4}\left\langle\cos ^{2} \theta\right\rangle \simeq \frac{G}{4} \cos ^{2} \theta_{\mathrm{H}}+a\langle\omega\rangle$, where $\theta_{\mathrm{H}}$ of the saddle point $\left(\theta_{\mathrm{H}}, 0\right)$ on the BDC branch in Fig. 3(b) is evaluated at the homoclinic bifurcation points and $a$ is a coefficient that needs to be determined numerically. Equation (41) can be approximated in the vicinity of the bifurcation points as

$$
\left\langle J_{Q_{\mathrm{b}}}\right\rangle=\frac{G}{4} \cos ^{2} \theta_{\mathrm{H}} \Delta T+\left(a+\frac{T_{\mathrm{eq}} n R r \sigma_{\mathrm{p}}}{2}\left\langle\frac{\sin ^{2} \theta}{V(\theta)}\right\rangle_{\theta}\right)\langle\omega\rangle .
$$

In Fig. 5(b), Eq. (42) is compared with the numerical results for the bifurcation point $\tilde{T}_{\text {load }}^{\prime}$. They are in good agreement and the linear decreasing from the constant value is confirmed.

\section{Quasilinear response regime}

The angular velocity $\langle\omega\rangle$ shows a linear dependency on $T_{\text {load }}$ as it deviates from the bifurcation point to a sufficient extent [Fig. 3(a)]. It also shows a similar linear dependency with respect to $\Delta T$ [6]. We call a regime with this linear dependency a quasilinear response regime. In this regime, we may approximate $\omega$ by a constant value $\Omega$ as $\omega \simeq \Omega$ by assuming that the periodic variation around the constant value is sufficiently small. Under this assumption, one cycle period is approximated as $d t \simeq \frac{d \theta}{\Omega}$ and thus $\tau=\int_{0}^{\tau} d t \simeq \int_{0}^{2 \pi} \frac{d \theta}{\Omega}=$ $\frac{2 \pi}{\Omega}$. Thus, the rotational torque component in Eq. (39) is approximated as

$$
\left\langle\sigma_{\mathrm{p}}\left(\frac{n R T_{\mathrm{eff}}(\theta)}{V(\theta)}-p_{\mathrm{air}}\right) r \sin \theta\right\rangle \simeq \frac{\sigma_{\mathrm{p}} n R r}{2}\left\langle\frac{\sin ^{2} \theta}{V(\theta)}\right\rangle_{\theta} \Delta T,
$$


where we have used $\int_{0}^{2 \pi} \frac{\sin \theta}{V(\theta)} d \theta=0$ and $\int_{0}^{2 \pi} p_{\text {air }} \sin \theta d \theta=0$. Then Eq. (39) is reduced to

$$
\Omega=\frac{\frac{\sigma_{\mathrm{p}} n R r}{2}\left\langle\frac{\sin ^{2} \theta}{V(\theta)}\right\rangle_{\theta} \Delta T-T_{\mathrm{load}}}{\Gamma+\frac{\sigma_{\mathrm{p}}^{2} n^{2} R^{2} T_{\mathrm{eq}} r^{2}}{G}\left\langle\frac{\sin ^{2} \theta}{V^{2}(\theta)}\right\rangle_{\theta}} .
$$

In Fig. 3(a), the theoretical line and numerical calculations are compared, and they are in good agreement.

Next, we consider the heat flux, Eq. (41), in the quasilinear response regime using the result of Eq. (44). By approximating $d t \simeq \frac{d \theta}{\Omega}$ as above, we have $\frac{G}{4}\left\langle\cos ^{2} \theta\right\rangle \simeq \frac{G}{4}\left\langle\cos ^{2} \theta\right\rangle_{\theta} \simeq \frac{G}{8}$. Then the heat flux in Eq. (41) is approximated as

$$
\begin{aligned}
\left\langle J_{Q_{\mathrm{b}}}\right\rangle \simeq & \frac{G}{8} \Delta T+\frac{T_{\mathrm{eq}} n R r \sigma_{\mathrm{p}}}{2}\left\langle\frac{\sin ^{2} \theta}{V(\theta)}\right\rangle_{\theta} \Omega \\
= & -\frac{\frac{T_{\mathrm{eq}} n R r \sigma_{\mathrm{p}}}{2}\left\langle\frac{\sin ^{2} \theta}{V(\theta)}\right\rangle_{\theta}}{\Gamma+\frac{\sigma_{\mathrm{p}}^{2} n^{2} R^{2} T_{\mathrm{eq}} r^{2}}{G}\left\langle\frac{\sin ^{2} \theta}{V^{2}(\theta)}\right\rangle_{\theta}} T_{\mathrm{load}} \\
& +\left(\frac{G}{8}+\frac{\frac{T_{\mathrm{eq}} n^{2} R^{2} r^{2} \sigma_{\mathrm{p}}^{2}}{4}\left\langle\frac{\sin ^{2} \theta}{V(\theta)}\right\rangle_{\theta}^{2}}{\Gamma+\frac{\sigma_{\mathrm{p}}^{2} n^{2} R^{2} T_{\mathrm{eq}} r^{2}}{G}\left\langle\frac{\sin ^{2} \theta}{V^{2}(\theta)}\right\rangle_{\theta}}\right) \Delta T,
\end{aligned}
$$

where we have used $\int_{0}^{2 \pi} \frac{\sin \theta}{V(\theta)} d \theta=0$. The theoretical line and numerical calculations show a good agreement [Fig. 5(a)]. Note that $\left\langle J_{Q_{\mathrm{t}}}\right\rangle \simeq-\left\langle J_{Q_{\mathrm{b}}}\right\rangle$, which can be confirmed by repeating the same calculations as $\left\langle J_{Q_{\mathrm{b}}}\right\rangle$. The theoretical expressions Eqs. (44) and (45) are used for developing a theory of the thermodynamic efficiency of the engine in the quasilinear response regime in $\mathrm{Sec}$. V.

\section{THEORY OF THERMODYNAMIC EFFICIENCY}

\section{A. Definition of power and thermodynamic efficiency}

We define the power and thermodynamic efficiency of the LTD Stirling engine. The instantaneous power produced by the gas, which is the second term on the right-hand side of Eq. (13), can be rewritten as

$$
\begin{aligned}
w & \equiv \frac{n R T}{V} \frac{d V}{d t} \\
& =\frac{n R T}{V}\left(r \sin \theta \sigma_{\mathrm{p}}\right) \omega \\
& =\left(I \frac{d \omega}{d t}+r \sigma_{\mathrm{p}} p_{\mathrm{air}} \sin \theta+\Gamma \omega+T_{\mathrm{load}}\right) \omega \\
& =\frac{d}{d t}\left(\frac{I}{2} \omega^{2}\right)+p_{\mathrm{air}} \frac{d V}{d t}+\Gamma \omega^{2}+T_{\mathrm{load}} \omega,
\end{aligned}
$$

where we have used Eq. (19) from the second line to the third line. We can interpret each term in Eq. (46) as follows. The first term is the rotational kinetic energy change of the crank, and the second, third, and last terms represent the work carried out against the atmospheric pressure, frictional torque, and load torque, respectively.
By using Eq. (46), we define the cycle-averaged power as

$$
\begin{aligned}
P \equiv\langle w\rangle=\frac{1}{\tau} \int_{0}^{\tau} w d t & =\frac{1}{\tau} \int_{0}^{\tau}\left(\Gamma \omega^{2}+T_{\mathrm{load}} \omega\right) d t \\
& =\Gamma \frac{1}{\tau} \int_{0}^{\tau} \omega^{2} d t+T_{\mathrm{load}}\left(\frac{2 \pi}{\tau}\right) \\
& =\Gamma\left\langle\omega^{2}\right\rangle+T_{\mathrm{load}}\langle\omega\rangle \\
& \equiv P_{\text {fric }}+P_{\mathrm{load}}
\end{aligned}
$$

where we have used $\int_{0}^{\tau} \frac{d}{d t}\left(\frac{I}{2} \omega^{2}\right) d t=0$ and $\int_{0}^{\tau} p_{\text {air }} \frac{d V}{d t} d t=0$. The power $P$, which is referred to as the indicated power [24], defined as the closed area of the pressure-volume diagram of an engine, was decomposed into that carried out against the friction torque $P_{\text {fric }}$ and that carried out against the load torque $P_{\text {load }}$, referred to as the brake power [24]. The former is eventually dissipated into the surrounding air as heat. By timeaveraging the energy conservation equation, Eq. (13), we have $\left\langle J_{Q_{\mathrm{b}}}\right\rangle+\left\langle J_{Q_{\mathrm{t}}}\right\rangle=P_{\text {load }}+P_{\text {fric }}$. The thermodynamic efficiency $\eta$ is then defined as the ratio of the input heat flux from the hot heat reservoir converted into the available power exerted against the load torque (brake power) $P_{\text {load }}$ [24]. For $\Delta T>0$, it is explicitly given as

$$
\eta \equiv \frac{P_{\text {load }}}{\left\langle J_{Q_{\mathrm{b}}}\right\rangle}=\frac{T_{\mathrm{load}}\langle\omega\rangle}{\left\langle J_{Q_{\mathrm{b}}}\right\rangle} .
$$

In Figs. 6(a) and 6(b), we present the numerical results of the $\tilde{T}_{\text {load }}$ dependence of the (nondimensionalized) brake power $\tilde{P}_{\text {load }}=\frac{P_{\text {load }}}{n R T_{\text {eq }} \sqrt{\frac{n R T_{\text {eq }}}{I}}}$ and the efficiency $\eta$, respectively. We can see that the values at which the maximum efficiency and maximum power are realized are close, which is characteristic of heat engines operating with nonnegligible heat leakage (the first term on the right-hand side of Eq. (41) for the present model) [26]. When the maximum efficiency is located in the quasilinear response regime, we can obtain its theoretical value, as we show in Sec. V B.

\section{B. Thermodynamic theory of an LTD kinematic Stirling heat engine in a quasilinear response regime}

Before constructing a thermodynamic theory of the LTD kinematic Stirling engine, we review the theory for conventional linear irreversible heat engines [9-11]. For generic heat engines, the entropy production rate of the total system $\dot{\sigma}$ (a heat engine and heat reservoirs at temperatures $T_{0}$ and $T_{1}$ ) is given by

$$
\dot{\sigma} \equiv-\frac{\dot{Q}_{0}}{T_{0}}-\frac{\dot{Q}_{1}}{T_{1}}=-\frac{P_{\mathrm{load}}}{T_{1}}+\dot{Q}_{0}\left(\frac{1}{T_{1}}-\frac{1}{T_{0}}\right),
$$

where $Q_{0}\left(Q_{1}\right)$ is the heat flowing into the working substance from the heat reservoir at $T_{0}\left(T_{1}\right)$, and we have used $P_{\text {load }}=$ $\dot{Q}_{0}+\dot{Q}_{1}$ (the energy conservation law). Hereafter, an overdot refers to quantities per unit time for steady-state heat engines or quantities averaged over one cycle period for cyclic heat engines. We can express $P_{\text {load }}$ as $P_{\text {load }}=F \dot{x}$ using an external force $F$ and its conjugate flux $\dot{x}$. By taking the limit of a small temperature difference and small external force, we can 

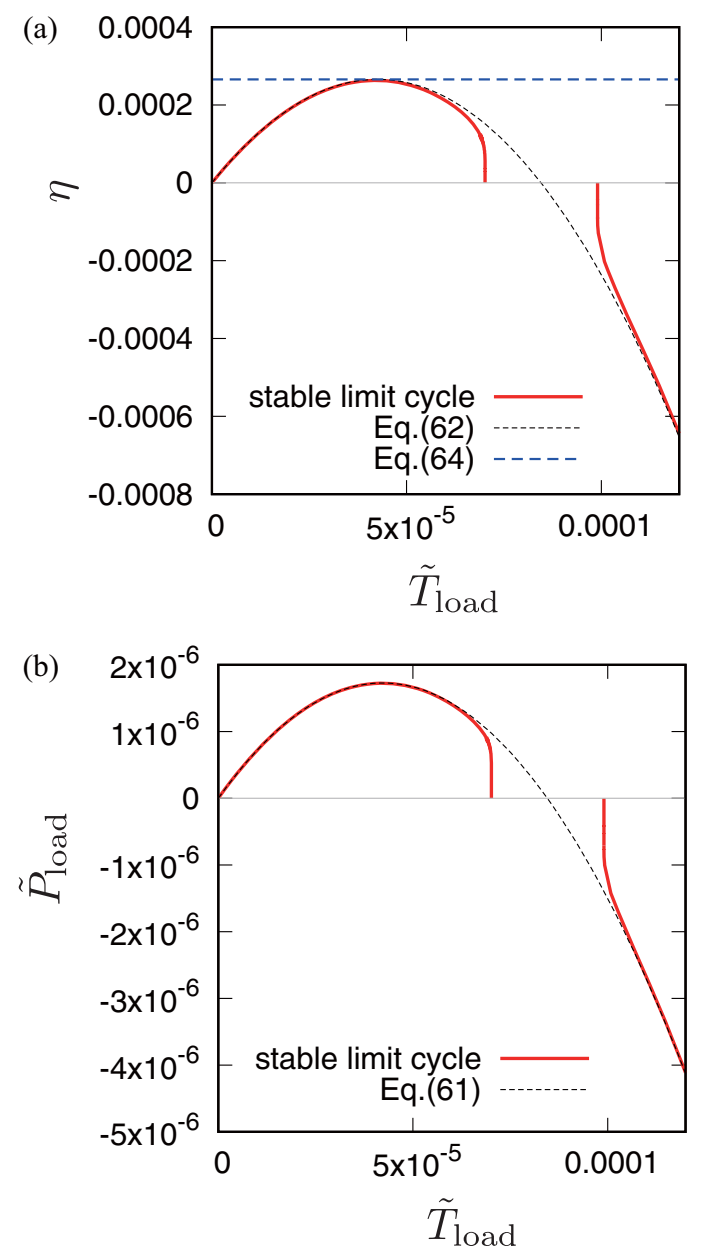

FIG. 6. (a) Thermodynamic efficiency $\eta$ in Eq. (48) and (b) (nondimensionalized) brake power $\tilde{P}_{\text {load }}$ in Eq. (47) as a function of the load torque $\tilde{T}_{\text {load }}$. Dashed curves denote the theoretical curves of Eqs. (61) and (62). The dashed line is the maximum efficiency given in Eq. (64) using $q \simeq 0.17513$.

approximate $\dot{\sigma}$ as

$$
\begin{aligned}
\dot{\sigma} & \simeq \dot{x}\left(-\frac{F}{T_{\mathrm{eq}}}\right)+\dot{Q}_{0} \frac{\Delta T}{T_{\mathrm{eq}}^{2}} \\
& =J_{1} F_{1}+J_{2} F_{2},
\end{aligned}
$$

where the temperature difference and the averaged temperature are given as $\Delta T=T_{0}-T_{1}$ and $T_{\mathrm{eq}}=\frac{T_{0}+T_{1}}{2}$, respectively, for the present setup. Here, we have defined the thermodynamic forces $F_{i}$ and their conjugate fluxes $J_{i}$ as

$$
J_{1} \equiv \dot{x}, \quad F_{1} \equiv-\frac{F}{T_{\text {eq }}}
$$

and

$$
J_{2} \equiv \dot{Q}_{0}, \quad F_{2} \equiv \frac{\Delta T}{T_{\mathrm{eq}}^{2}} .
$$

In linear irreversible thermodynamics, we assume the following linear relations between the thermodynamic fluxes and forces as

$$
\begin{aligned}
& J_{1}=L_{11} F_{1}+L_{12} F_{2}, \\
& J_{2}=L_{21} F_{1}+L_{22} F_{2},
\end{aligned}
$$

where $L_{i j}$ are the Onsager coefficients with reciprocity $L_{12}=$ $L_{21}[21,22]$. The use of Eqs. (53) and (54) enables us to rewrite Eq. (50) as

$$
\dot{\sigma}=L_{11} F_{1}^{2}+2 L_{12} F_{1} F_{2}+L_{22} F_{2}^{2} .
$$

From $\dot{\sigma} \geqslant 0$ for the arbitrary $F_{1}$ and $F_{2}$ (the second law of thermodynamics), we obtain the following restrictions on the Onsager coefficients $L_{i j}$ :

$$
L_{11} \geqslant 0, \quad L_{22} \geqslant 0, \quad L_{11} L_{22}-L_{12} L_{21} \geqslant 0 .
$$

Here, we define the coupling-strength parameter $q$ as

$$
q \equiv \frac{L_{12}}{\sqrt{L_{11} L_{22}}},
$$

which should satisfy $|q| \leqslant 1$ from the last inequality in Eq. (56). The meaning of $q$ can be elucidated by rewriting the heat flux in Eq. (54) by using $J_{1}$ instead of $F_{1}$ as

$$
J_{2}=\frac{L_{21}}{L_{11}} J_{1}+L_{22}\left(1-q^{2}\right) F_{2} .
$$

The case of $|q|=1$ is an ideal condition known as the tightcoupling condition for which the heat flux $J_{2}$ is in proportion to the motion flux $J_{1}$. For the non-tight-coupling case $|q| \neq 1$, the nonvanishing heat leakage $L_{22}\left(1-q^{2}\right) F_{2}$ arises from the simultaneous contact between the two heat reservoirs on the engine, which decreases the thermodynamic performance of the engine, as shown below.

The power and thermodynamic efficiency are written using the thermodynamic fluxes and forces in Eqs. (53) and (54) as

$$
\begin{aligned}
& P_{\text {load }}=F \dot{x}=-J_{1} F_{1} T_{\mathrm{eq}}, \\
& \eta=\frac{P_{\text {load }}}{\dot{Q}_{0}}=-\frac{J_{1} F_{1} T_{\mathrm{eq}}}{J_{2}},
\end{aligned}
$$

respectively, where we assume $F_{2}>0$. It is more convenient to express them in terms of $J_{1}$ instead of $F_{1}$ as

$$
\begin{gathered}
P_{\text {load }}=\frac{L_{12}}{L_{11}} J_{1} F_{2} T_{\text {eq }}-\frac{T_{\text {eq }}}{L_{11}} J_{1}^{2}, \\
\eta=\frac{\frac{L_{12}}{L_{11}} J_{1} F_{2} T_{\text {eq }}-\frac{T_{\text {eq }}}{L_{11}} J_{1}^{2}}{\frac{L_{21}}{L_{11}} J_{1}+L_{22}\left(1-q^{2}\right) F_{2}},
\end{gathered}
$$

using Eqs. (53) and (58). For the tight-coupling case $|q|=$ 1 , the quasistatic limit $J_{1} \rightarrow 0$ yields the vanishing power $P_{\text {load }} \rightarrow 0$ and the Carnot efficiency $\eta \rightarrow F_{2} T_{\text {eq }}=\frac{\Delta T}{T_{\text {eq }}}=$ $\frac{\Delta T}{T_{0}-\frac{\Delta T}{2}} \simeq \frac{\Delta T}{T_{0}} \equiv \eta_{\mathrm{C}}$. For the non-tight-coupling case $|q| \neq 1$, $J_{1}$ that yields the maximum efficiency is obtained as the solution of $\frac{\partial \eta}{\partial J_{1}}=0$,

$$
J_{1}^{\max }=\frac{L_{21}\left(1-q^{2}\right) F_{2}}{q^{2}}\left\{-1+\sqrt{\frac{1}{1-q^{2}}}\right\},
$$

which takes a finite value, unlike the quasistatic limit $J_{1} \rightarrow 0$ for the tight-coupling case $|q|=1$. The maximum efficiency then reads $[10,11]$

$$
\eta_{\max }=\frac{\left(1-\sqrt{1-q^{2}}\right)^{2}}{q^{2}} \eta_{\mathrm{C}}
$$

which is a monotonic function of $q$. 
The efficiency at maximum power $\eta^{*}$ attained at $J_{1}^{*}=$ $\frac{L_{12}}{2} F_{2}$ is also given as [9]

$$
\eta^{*}=\frac{1}{2} \frac{q^{2}}{2-q^{2}} \eta_{\mathrm{C}}
$$

For the tight-coupling case $|q|=1$, we obtain $\eta^{*}=\frac{\eta_{\mathrm{C}}}{2}$ (the Curzon-Ahlborn efficiency [18]) as the upper bound.

Thus far, we have reviewed the theory for conventional linear irreversible heat engines. Returning to our model of the LTD kinematic Stirling engine, the linear response relations such as Eqs. (53) and (54) expanded from an equilibrium state with $F_{1}=0$ and $F_{2}=0$ do not hold. This is because the rotational state described as the limit cycle is not connected to the equilibrium state, and the linear dependency in Eqs. (44) and (45) holds only when the external forces deviate sufficiently far from the bifurcation points. Nevertheless, we can formally write the linear relations applied to these quasilinear response regimes in terms of the thermodynamic fluxes and forces.

We identify each quantity used in the theory of the linear irreversible heat engines as $T_{0}=T_{\mathrm{b}}, T_{1}=T_{\mathrm{t}}, \dot{x}=\Omega, F=T_{\text {load }}$, $\dot{Q}_{0}=\left\langle J_{Q_{b}}\right\rangle$, and $\dot{Q}_{1}=\left\langle J_{Q_{\mathrm{t}}}\right\rangle-P_{\text {fric }}$. Using these quantities, we can write the entropy production rate of the LTD kinematic Stirling engine in the quasilinear response regime as

$$
\begin{aligned}
\dot{\sigma}=-\frac{\left\langle J_{Q_{\mathrm{b}}}\right\rangle}{T_{\mathrm{b}}}-\frac{\left\langle J_{Q_{\mathrm{t}}}\right\rangle-P_{\text {fric }}}{T_{\mathrm{t}}} & =-\frac{P_{\text {load }}}{T_{\mathrm{t}}}+\left\langle J_{Q_{\mathrm{b}}}\right\rangle\left(\frac{1}{T_{\mathrm{t}}}-\frac{1}{T_{\mathrm{b}}}\right) \\
& \simeq \Omega\left(-\frac{T_{\text {load }}}{T_{\text {eq }}}\right)+\left\langle J_{Q_{\mathrm{b}}}\right\rangle \frac{\Delta T}{T_{\text {eq }}^{2}} \\
& =J_{1} F_{1}+J_{2} F_{2},
\end{aligned}
$$

where we have used $P_{\text {load }}=\left\langle J_{Q_{\mathrm{b}}}\right\rangle+\left\langle J_{Q_{\mathrm{t}}}\right\rangle-P_{\text {fric }}$ (the energy conservation law), and the thermodynamic fluxes and forces are related via the linear relations

$$
\begin{aligned}
& J_{1}=L_{11}^{\prime} F_{1}+L_{12}^{\prime} F_{2}, \\
& J_{2}=L_{21}^{\prime} F_{1}+L_{22}^{\prime} F_{2},
\end{aligned}
$$

where $L_{i j}^{\prime}$ are the quasilinear response coefficients. The prime notation is used to demonstrate that they are defined for the quasilinear response regime. The use of the definitions of the thermodynamic fluxes and forces, and Eqs. (44) and (45), makes it possible to identify the quasilinear response coefficients $L_{i j}^{\prime}$ as

$$
\begin{aligned}
& L_{i j}^{\prime}=\left(\begin{array}{ll}
L_{11}^{\prime} & L_{12}^{\prime} \\
L_{21}^{\prime} & L_{22}^{\prime}
\end{array}\right)
\end{aligned}
$$

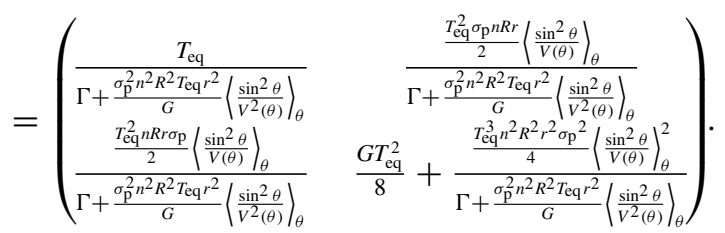

Here, we can confirm that a symmetric relation holds as $L_{12}^{\prime}=$ $L_{21}^{\prime}$. In Fig. 7, the quasilinear response coefficients in Eq. (69) and the symmetric relation are numerically confirmed. At this point, the origin of this symmetry is not questioned and it is elucidated in Sec. VI in terms of the (anti-)reciprocity
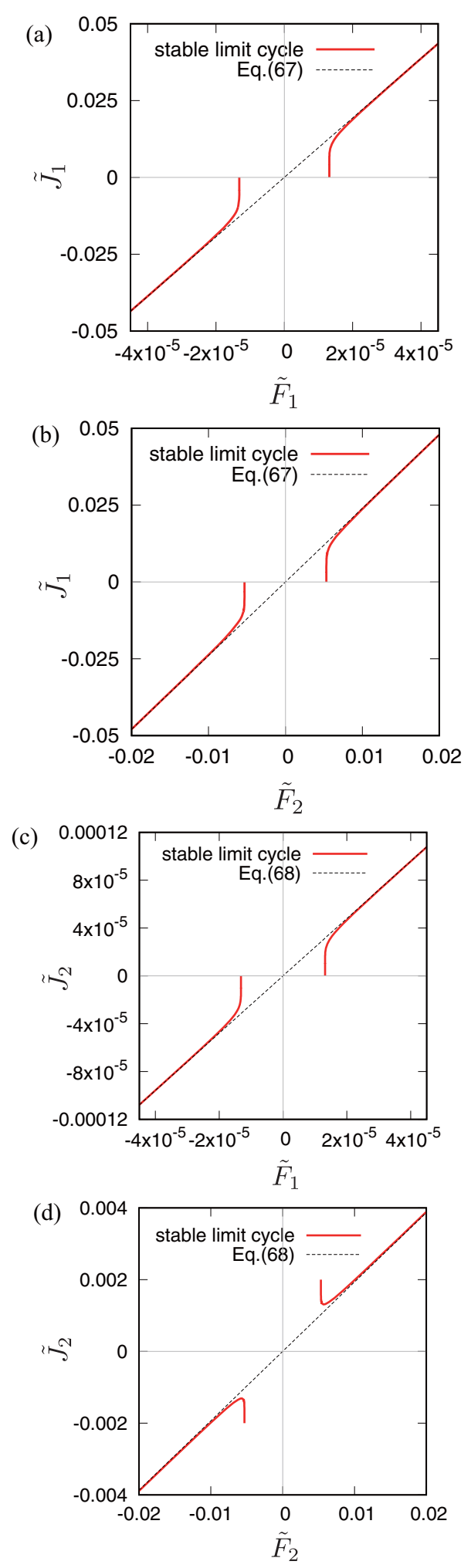

FIG. 7. Relations between the (nondimensionalized) thermodynamic fluxes $\tilde{J}_{i}$ and forces $\tilde{F}_{j}$, where $\tilde{F}_{1}=-\tilde{T}_{\text {load }}$ and $\tilde{F}_{2}=\Delta \tilde{T}$. (a) $\tilde{J}_{1}-\tilde{F}_{1}$ diagram for $\tilde{F}_{2}=0$, (b) $\tilde{J}_{1}-\tilde{F}_{2}$ diagram for $\tilde{F}_{1}=0$, (c) $\tilde{J}_{2}-\tilde{F}_{1}$ diagram for $\tilde{F}_{2}=0$, and (d) $\tilde{J}_{2}-\tilde{F}_{2}$ diagram for $\tilde{F}_{1}=0$. Dashed lines denote Eqs. (67) and (68) using the quasilinear response coefficients $\tilde{L}_{i j}^{\prime}$ in Eq. (69). In (b) and (c), the symmetric relation $\tilde{L}_{12}^{\prime}=\tilde{L}_{21}^{\prime}$ is observed. 
of the Onsager kinetic coefficients. Because the quasilinear response relations, Eqs. (67) and (68), with the symmetric relation formally take the same form as the conventional Onsager relations, Eqs. (53) and (54), the thermodynamic theory developed using Eqs. (53) and (54) is also applied to the quasilinear response regime.

In the present case, the coupling-strength parameter $q$ in Eq. (57) is calculated from the quasilinear response coefficients in Eq. (69) as

$$
\begin{aligned}
q & =\frac{1}{\sqrt{1+\frac{1}{2} \frac{\left\langle\frac{\sin ^{2} \theta}{V^{2}(\theta)}\right\rangle_{\theta}}{\left\langle\frac{\sin ^{2} \theta}{V(\theta)}\right\rangle_{\theta}^{2}}+\frac{G \Gamma}{2 T_{\mathrm{eq}} n^{2} R^{2} r^{2} \sigma_{\mathrm{p}}^{2}\left\langle\frac{\sin ^{2} \theta}{V(\theta)}\right\rangle_{\theta}^{2}}}} \\
& =\frac{1}{\sqrt{1+\frac{1}{2} \frac{\left\langle\frac{\sin ^{2} \theta}{\tilde{V}^{2}(\theta)}\right\rangle_{\theta}}{\left\langle\frac{\sin ^{2} \theta}{\tilde{V}(\theta)}\right\rangle_{\theta}^{2}}+\frac{\tilde{G} \tilde{\Gamma}}{2 \tilde{\sigma}^{2}\left\langle\frac{\sin ^{2} \theta}{\tilde{V}(\theta)}\right\rangle_{\theta}^{2}}}}
\end{aligned}
$$

Notably, the coupling strength depends on three major (nondimensionalized) physical parameters of the model: $\tilde{\sigma}, \tilde{G}$, and $\tilde{\Gamma}$. Thus, the maximum efficiency is given by Eq. (64), with the coupling strength $q$ in Eq. (70) being the single figure of merit.

In Figs. 6(a) and 6(b), we compare the numerical results of the efficiency and power with the theoretical results, Eqs. (62) and (61), using $L_{i j}^{\prime}$ in Eq. (69). We can find that the theory approximates the numerical results well. Although we used Eq. (61) for the calculations of $P_{\text {load }}$, it should be consistent with the expression $P_{\text {load }}=\left\langle J_{Q_{\mathrm{b}}}\right\rangle+\left\langle J_{Q_{\mathrm{t}}}\right\rangle-P_{\text {fric }}$ (the energy conservation law), which was used in the derivation of Eq. (66). See Appendix C for a detailed demonstration of the equivalence of these two expressions. The maximum efficiency in Eq. (64) using $q \simeq 0.17513$ calculated for the present parameters also approximates the numerical result well [Fig. 6(a)].

The simple formula, Eq. (64), using Eq. (70) may provide a new guiding principle for designing efficient LTD kinematic Stirling engines. By noting

$$
\begin{aligned}
& \left\langle\frac{\sin ^{2} \theta}{\tilde{V}(\theta)}\right\rangle_{\theta}=\frac{(1-\sqrt{1+\tilde{\sigma}})^{2}}{\tilde{\sigma}^{2}}, \\
& \left\langle\frac{\sin ^{2} \theta}{\tilde{V}^{2}(\theta)}\right\rangle_{\theta}=\frac{(1-\sqrt{1+\tilde{\sigma}})^{2}}{2 \tilde{\sigma}^{2} \sqrt{1+\tilde{\sigma}}},
\end{aligned}
$$

we obtain $q \rightarrow \frac{1}{\sqrt{2}}$ as the upper bound of $q$ in Eq. (70) as $\tilde{\sigma} \rightarrow 0$ and $\tilde{G} \tilde{\Gamma} \rightarrow 0$, with $\tilde{G} \tilde{\Gamma} \ll \tilde{\sigma}^{2}$ being satisfied. Within this limit, $\eta_{\max }$ in Eq. (64) is given as

$$
\lim _{q \rightarrow \frac{1}{\sqrt{2}}} \eta_{\max }=(3-2 \sqrt{2}) \eta_{\mathrm{C}} \approx 0.17157 \eta_{\mathrm{C}} .
$$

This is the upper bound that the present model in the quasilinear response regime can attain. We note that $\eta_{\max }$ of the present model cannot attain the Carnot efficiency achieved by the ideal Stirling cycle because it lacks a regenerator.

We can also obtain

$$
\lim _{q \rightarrow \frac{1}{\sqrt{2}}} \eta^{*}=\frac{1}{6} \eta_{\mathrm{C}}
$$

as the upper bound of the efficiency at maximum power in Eq. (65) that the present model in the quasilinear response regime can attain.

\section{ORIGIN OF THE SYMMETRIC RELATION}

The symmetric relation $L_{12}^{\prime}=L_{21}^{\prime}$ in Eq. (69) is reminiscent of the Onsager reciprocity in linear irreversible thermodynamics, whereas the rotational state of the engine described as the limit cycle may not be described as a linear response regime. Here, we explain the origin of the symmetry in terms of the (anti-)reciprocity of the Onsager kinetic coefficients $[21,22,27]$ in the original three-dimensional dynamical model, Eqs. (14)-(16), before the adiabatic elimination.

\section{A. Relaxation dynamics towards the equilibrium state}

Let us consider that a mesoscopic LTD Stirling heat engine specified by $\left(\theta, p_{\theta}, U\right)$ is in thermal equilibrium with a heat reservoir, where $p_{\theta} \equiv I \omega$ is the angular momentum of the crank and $U=\frac{f}{2} n R T$ is the internal energy of the gas. The engine may be perturbed from the equilibrium state $\left(\theta_{\mathrm{eq}}, 0, U_{\mathrm{eq}}\right)$ by thermal fluctuation and relaxes to the original equilibrium state, where $U_{\text {eq }}=\frac{f}{2} n R T_{\text {eq. }}$. By linearizing Eqs. (14)-(16) with $\Delta T=0$ and $T_{\text {load }}=0$ around the equilibrium value, we obtain the following linear relaxation equations:

$$
\begin{gathered}
\frac{d \delta \theta}{d t}=\frac{1}{I} \delta p_{\theta} \\
\frac{d \delta p_{\theta}}{d t}=-\frac{n R r^{2} \sigma_{\mathrm{p}}^{2} T_{\mathrm{eq}} \sin ^{2} \theta_{\mathrm{eq}}}{V^{2}\left(\theta_{\mathrm{eq}}\right)} \delta \theta-\frac{\Gamma}{I} \delta p_{\theta}+r \sigma_{\mathrm{p}} \frac{2 \sin \theta_{\mathrm{eq}}}{f V\left(\theta_{\mathrm{eq}}\right)} \delta U \\
\frac{d \delta U}{d t}=-\frac{n R T_{\mathrm{eq}} r \sigma_{\mathrm{p}} \sin \theta_{\mathrm{eq}}}{I V\left(\theta_{\mathrm{eq}}\right)} \delta p_{\theta}-\frac{2 G}{f n R} \delta U
\end{gathered}
$$

These are rewritten as $(k, l=\theta, p, U)$

$$
\frac{d x_{k}}{d t}=-\lambda_{k l} x_{l},
$$

where $x_{\theta} \equiv \delta \theta, x_{p} \equiv \delta p_{\theta}$, and $x_{U} \equiv \delta U$ are the thermodynamic variables that express variation (or fluctuation) from the equilibrium state, and $\lambda_{k l}$ are the linear relaxation coefficients. Next, we express Eq. (78) [Eqs. (75)-(77)] as

$$
\frac{d x_{k}}{d t}=-\gamma_{k l} X_{l},
$$

where $X_{l}$ are the conjugate thermodynamic forces to be determined, and $\gamma_{k l}$ are the Onsager kinetic coefficients.

Following the methods in [27], we introduce $\delta H$ as the change in the crank's Hamiltonian from the vanishing value at the equilibrium state $\left(\theta_{\mathrm{eq}}, 0, U_{\mathrm{eq}}\right)$ as

$$
\delta H=\frac{\delta p_{\theta}^{2}}{2 I}+\frac{n R r^{2} \sigma_{\mathrm{p}}^{2} T_{\mathrm{eq}} \sin ^{2} \theta_{\mathrm{eq}}}{V^{2}\left(\theta_{\mathrm{eq}}\right)} \frac{\delta \theta^{2}}{2} .
$$

We then define $X_{\theta}$ and $X_{p}$ as the thermodynamic forces for the mechanical degrees of freedom as

$$
\begin{gathered}
X_{\theta}=\frac{1}{T_{\mathrm{eq}}} \frac{\partial \delta H}{\partial x_{\theta}}=\frac{n R r^{2} \sigma_{\mathrm{p}}^{2} \sin ^{2} \theta_{\mathrm{eq}}}{V^{2}\left(\theta_{\mathrm{eq}}\right)} \delta \theta, \\
X_{p}=\frac{1}{T_{\mathrm{eq}}} \frac{\partial \delta H}{\partial x_{p}}=\frac{1}{T_{\mathrm{eq}}} \frac{\delta p_{\theta}}{I},
\end{gathered}
$$


where we can interpret $X_{\theta}$ as a restoring force and $X_{p}$ as an inertial force. Under these thermodynamic forces, we can easily find

$$
\begin{gathered}
\gamma_{\theta p}=-T_{\mathrm{eq}}, \\
\gamma_{p \theta}=T_{\mathrm{eq}},
\end{gathered}
$$

which satisfy the Onsager's antireciprocal relation $\gamma_{\theta p}=$ $-\gamma_{p \theta}$. We note that the antireciprocity is fundamentally derived from the fact that $x_{\theta}$ is a time-reversely symmetric quantity, whereas $x_{p}$ is an antisymmetric quantity under time reversal of microscopic dynamics [27]. We also find

$$
\begin{gathered}
\gamma_{p p}=T_{\mathrm{eq}} \Gamma, \\
\gamma_{U p}=\gamma_{U p}\left(\theta_{\mathrm{eq}}\right)=\frac{n R T_{\mathrm{eq}}^{2} r \sigma_{\mathrm{p}} \sin \theta_{\mathrm{eq}}}{V\left(\theta_{\mathrm{eq}}\right)} .
\end{gathered}
$$

Once $X_{\theta}$ and $X_{p}$ have been determined as above, $X_{U}$, the other thermodynamic force of the thermodynamic degree of freedom, can be uniquely determined such that it satisfies the Onsager symmetry principle [27]. Because we want to have the antireciprocal relation $\gamma_{U p}=-\gamma_{p U}$ for $x_{U}$ as a timereversely symmetric quantity, we naturally choose $X_{U}$ as

$$
X_{U}=\frac{\delta T}{T_{\mathrm{eq}}^{2}},
$$

which determines

$$
\begin{aligned}
\gamma_{p U}=\gamma_{p U}\left(\theta_{\mathrm{eq}}\right) & =-\frac{n R T_{\mathrm{eq}}^{2} r \sigma_{\mathrm{p}} \sin \theta_{\mathrm{eq}}}{V\left(\theta_{\mathrm{eq}}\right)}, \\
\gamma_{U U} & =G T_{\mathrm{eq}}^{2} .
\end{aligned}
$$

The other kinetic coefficients vanish as $\gamma_{\theta \theta}=\gamma_{\theta U}=\gamma_{U \theta}=0$.

We note that the thermodynamic variables $x_{l}$ and the forces $X_{k}$ are linearly related from Eqs. (78) and (79) as

$$
X_{k}=\beta_{k l} x_{l},
$$

where

$$
\beta_{k l} \equiv \gamma_{k m}^{-1} \lambda_{m l}
$$

The only nonvanishing components of $\beta_{k l}$ are the diagonal elements as

$$
\begin{gathered}
\beta_{\theta \theta}=\frac{n R r^{2} \sigma_{\mathrm{p}}^{2} \sin ^{2} \theta_{\mathrm{eq}}}{V^{2}\left(\theta_{\mathrm{eq}}\right)}, \\
\beta_{p p}=\frac{1}{T_{\mathrm{eq}} I}, \\
\beta_{U U}=\frac{2}{f n R T_{\mathrm{eq}}^{2}} .
\end{gathered}
$$

The total entropy variation (the heat engine and reservoir) around the maximum, equilibrium value is then approximated as the following quadratic form [27]:

$$
\begin{aligned}
\delta S & =-\frac{1}{2} \beta_{k l} x_{k} x_{l} \\
& =-\frac{\delta p_{\theta}^{2}}{2 I T_{\mathrm{eq}}}-\frac{n R r^{2} \sigma_{\mathrm{p}}^{2} \sin ^{2} \theta_{\mathrm{eq}}}{V^{2}\left(\theta_{\mathrm{eq}}\right)} \frac{\delta \theta^{2}}{2}-\frac{f n R}{4} \frac{\delta T^{2}}{T_{\mathrm{eq}}^{2}} .
\end{aligned}
$$

This variation is consistent with equilibrium statistical mechanics; the fluctuation of the temperature and volume of a system under isothermal and isobaric conditions obeys the probability distribution [27]

$$
w(\delta T, \delta V) \propto \exp \left\{-\frac{C_{V}}{2 k_{\mathrm{B}} T_{\mathrm{eq}}^{2}}(\delta T)^{2}+\frac{1}{2 k_{\mathrm{B}} T_{\mathrm{eq}}}\left(\frac{\partial p}{\partial V}\right)_{T_{\mathrm{eq}}}(\delta V)^{2}\right\},
$$

where $C_{V}$ and $k_{\mathrm{B}}$ are the constant-volume heat capacity of the system and the Boltzmann constant, respectively. The momentum of the system also fluctuates around its equilibrium value $\delta p_{\theta}=0$ according to the Maxwell distribution:

$$
w\left(\delta p_{\theta}\right) \propto \exp \left(-\frac{\delta p_{\theta}^{2}}{2 I k_{\mathrm{B}} T_{\mathrm{eq}}}\right) .
$$

According to Einstein's fluctuation formula, the total weight $W=w(\delta T, \delta V) w\left(\delta p_{\theta}\right)$ is given using the entropy variation $\delta S$ as

$$
W \propto \exp \left(\frac{\delta S}{k_{\mathrm{B}}}\right)
$$

By noting $C_{V}=\frac{f}{2} n R,\left(\frac{\partial p}{\partial V}\right)_{T_{\mathrm{eq}}}=-\frac{n R T_{\mathrm{eq}}}{V^{2}}$, and $\delta V=\frac{\partial V}{\partial \theta} \delta \theta=$ $r \sigma_{\mathrm{p}} \sin \theta \delta \theta$ in Eq. (96), we find that $\delta S$ in Eq. (98) agrees with that in Eq. (95). We note that we can define the thermodynamic force using $\delta S$ as

$$
X_{k}=-\frac{\partial \delta S}{\partial x_{k}} .
$$

The instantaneous entropy production rate is thus given as

$$
\frac{d \delta S}{d t}=\frac{\partial \delta S}{\partial x_{k}} \frac{d x_{k}}{d t}=-X_{k} \frac{d x_{k}}{d t}=\gamma_{k l} X_{k} X_{l}=\gamma_{p p} X_{p}^{2}+\gamma_{U U} X_{U}^{2},
$$

to which the terms with the antireciprocal coefficients of $\gamma_{k l}$ do not contribute. Due to the antireciprocal component, the relaxation dynamics in the vicinity of the equilibrium state show damping oscillation toward the equilibrium state [28].

\section{B. Expression of quasilinear response coefficients using the Onsager kinetic coefficients}

Equations (75)-(77) describe the relaxation dynamics when the engine slightly deviates from the equilibrium state. For a nonequilibrium condition in which the externally sustained thermodynamic forces $\Delta T \neq 0$ and $T_{\text {load }} \neq 0$ are applied, the situation can drastically change. The engine can show rotational motion, and an engine under this state cannot be regarded as being in the linear response regime as we have seen in Sec. IV. Nevertheless, we examine how the antireciprocity of the Onsager kinetic coefficients $\gamma_{k l}$ included in the relaxation dynamics is inherited by the symmetric response coefficients $L_{i j}^{\prime}$.

We recall that we have adiabatically eliminated $T$ from the three-dimensional dynamical model, Eqs. (14)-(16), by assuming that the dynamics of the gas are fully subject to those of the crank, and obtained the two-dimensional dynamical model, Eqs. (18) and (19). The quasilinear relations in Sec. IV have been formulated for the rotational state of the two-dimensional dynamical model with $\Delta T \neq 0$ and $T_{\text {load }} \neq$ 0 . We now rewrite the thermodynamic fluxes $J_{1}=\Omega=\left\langle\frac{p_{\theta}}{I}\right\rangle$ 
and $J_{2}=\left\langle J_{Q_{\mathrm{b}}}\right\rangle$ of the two-dimensional model in a form that highlights the relation to the Onsager kinetic coefficients $\gamma_{k l}$ included in the relaxation dynamics of the three-dimensional dynamical model for $\Delta T=0$ and $T_{\text {load }}=0$.

For the rotational state realized under the nonequilibrium condition, the engine is largely perturbed from the equilibrium state $\left(\theta_{\mathrm{eq}}, 0, U_{\mathrm{eq}}\right)$. This is, however, only with respect to the phase angle $\theta$. Because the deviations of $p_{\theta}$ and $U$ (or, equivalently, $T$ ) from their equilibrium values are small even for the rotational state for small $|\Delta T|$ and $\left|T_{\text {load }}\right|$, we write $p_{\theta} \simeq \delta p_{\theta}$ and $T \simeq T_{\text {eq }}+\delta T$. We can thus expand Eq. (16) in terms of $\delta p_{\theta}$ and $\delta T$ around their equilibrium value $\left(p_{\theta}, T\right)=\left(0, T_{\mathrm{eq}}\right)$, with $\theta$ being held fixed as an arbitrary value,

$$
\frac{d \delta U}{d t}=G\left(\chi_{\mathrm{b}}(\theta)-\frac{1}{2}\right) \Delta T-\frac{n R T_{\mathrm{eq}} r \sigma_{\mathrm{p}} \sin \theta}{V(\theta)} \frac{\delta p_{\theta}}{I}-G \delta T .
$$

Equivalently, we have

$$
\frac{d x_{U}}{d t}=G\left(\chi_{\mathrm{b}}(\theta)-\frac{1}{2}\right) \Delta T-\gamma_{U p}(\theta) X_{p}-\gamma_{U U} X_{U},
$$

where we have denoted by $\gamma_{k l}(\theta)$ the Onsager kinetic coefficients, with $\theta_{\text {eq }}$ being formally replaced with $\theta$ of the stable limit cycle $(\theta, \omega)$. For $\Delta T=0$ and $T_{\text {load }}=0$, no stable limit cycle exists and Eq. (102) recovers the relaxation dynamics, Eq. (77), with $\theta=\theta_{\text {eq. }}$. The adiabatic approximation solution $T\left(\theta, \delta p_{\theta}\right)=T_{\text {eq }}+\delta T\left(\theta, \delta p_{\theta}\right)$ of Eq. (101) satisfying $\frac{d T}{d t}=$ $\frac{d \delta T}{d t}=0$ is given as

$\delta T\left(\theta, \delta p_{\theta}\right) \simeq\left(\chi_{\mathrm{b}}(\theta)-\frac{1}{2}\right) \Delta T-\frac{n R T_{\mathrm{eq}} r \sigma_{\mathrm{p}} \sin \theta}{G V(\theta)} \frac{\delta p_{\theta}}{I}$.

Equivalently, from Eq. (102), we have the adiabatic approximation solution as

$$
X_{U}=\left(\chi_{\mathrm{b}}(\theta)-\frac{1}{2}\right) F_{2}-\frac{\gamma_{U p}(\theta)}{\gamma_{U U}} X_{p},
$$

using the Onsager kinetic coefficients. We next expand Eq. (15), which describes the rotational state in terms of $\delta p_{\theta}$ and $\delta T$ around their equilibrium value $\left(p_{\theta}, T\right)=\left(0, T_{\mathrm{eq}}\right)$, with $\theta$ being held fixed as an arbitrary value as

$$
\begin{aligned}
\frac{d \delta p_{\theta}}{d t} & =\sigma_{\mathrm{p}}\left(\frac{n R\left(T_{\mathrm{eq}}+\delta T\right)}{V(\theta)}-p_{\text {air }}\right) r \sin \theta-\frac{\Gamma}{I} \delta p_{\theta}-T_{\text {load }} \\
& =\sigma_{\mathrm{p}}\left(\frac{n R T_{\mathrm{eq}}}{V(\theta)}-p_{\text {air }}\right) r \sin \theta-\frac{\Gamma}{I} \delta p_{\theta}
\end{aligned}
$$

$$
+\sigma_{\mathrm{p}} \frac{n R T_{\mathrm{eq}}^{2} r \sin \theta}{V(\theta)} \frac{\delta T}{T_{\mathrm{eq}}^{2}}-T_{\mathrm{load}}
$$

Equivalently, we can rewrite Eq. (105) as

$$
\frac{d x_{p}}{d t}=\sigma_{\mathrm{p}}\left(\frac{n R T_{\mathrm{eq}}}{V(\theta)}-p_{\text {air }}\right) r \sin \theta-\gamma_{p p} X_{p}-\gamma_{p U}(\theta) X_{U}+T_{\mathrm{eq}} F_{1}
$$

in terms of the thermodynamic forces. By putting $X_{U}$ in Eq. (104) into that in Eq. (106), we obtain

$$
\begin{aligned}
\frac{d x_{p}}{d t}= & \sigma_{\mathrm{p}}\left(\frac{n R T_{\mathrm{eq}}}{V(\theta)}-p_{\mathrm{air}}\right) r \sin \theta-\gamma_{p U}(\theta)\left(\chi_{\mathrm{b}}(\theta)-\frac{1}{2}\right) F_{2} \\
& +\left(\frac{\gamma_{p U}(\theta) \gamma_{U p}(\theta)}{\gamma_{U U}}-\gamma_{p p}\right) X_{p}+T_{\mathrm{eq}} F_{1} .
\end{aligned}
$$

Alternatively, by noting that $T_{\mathrm{b}}-T\left(\theta, \delta p_{\theta}\right)=\frac{\Delta T}{2}-$ $\delta T\left(\theta, \delta p_{\theta}\right)$ and using Eq. (103), we can also rewrite the instantaneous heat flux $J_{Q_{\mathrm{b}}}=G_{\mathrm{b}}(\theta)\left(T_{\mathrm{b}}-T\right)$ as

$$
\begin{aligned}
J_{Q_{\mathrm{b}}} & =G_{\mathrm{b}}(\theta)\left(\frac{\Delta T}{2}-\delta T\right) \\
& =G \chi_{\mathrm{b}}(\theta)\left(\chi_{\mathrm{t}}(\theta) T_{\mathrm{eq}}^{2} F_{2}+\frac{\gamma_{U p}(\theta)}{\gamma_{U U}} T_{\mathrm{eq}}^{2} X_{p}\right) \\
& =G T_{\mathrm{eq}}^{2} \chi_{\mathrm{b}}(\theta) \chi_{\mathrm{t}}(\theta) F_{2}+\chi_{\mathrm{b}}(\theta) \gamma_{U p}(\theta) X_{p} .
\end{aligned}
$$

We assume that the angular velocity $\frac{\delta p_{\theta}}{I}$ in the quasilinear response regime is a constant as $\frac{\delta p_{\theta}}{I}=T_{\mathrm{eq}} X_{p} \simeq \Omega$, in a manner similar to that we have assumed in Sec. IV D. By taking a time average of Eqs. (107) and (108), and repeating essentially the same calculations as in Sec. IV D, we have

$$
\begin{gathered}
J_{1}=\Omega=\left\langle\frac{\delta p_{\theta}}{I}\right\rangle=\frac{-T_{\mathrm{eq}}^{2} F_{1}+T_{\mathrm{eq}}\left\langle\gamma_{p U}(\theta) \chi_{\mathrm{b}}(\theta)\right\rangle_{\theta} F_{2}}{\frac{\left\langle\gamma_{p U}(\theta) \gamma_{U p}(\theta)\right\rangle_{\theta}}{\gamma_{U U}}-\gamma_{p p}}, \\
J_{2}=\left\langle J_{Q_{\mathrm{b}}}\right\rangle=G T_{\mathrm{eq}}^{2}\left\langle\chi_{\mathrm{b}}(\theta) \chi_{\mathrm{t}}(\theta)\right\rangle_{\theta} F_{2}+\left\langle\gamma_{U p}(\theta) \chi_{\mathrm{b}}(\theta)\right\rangle_{\theta} \frac{J_{1}}{T_{\mathrm{eq}}} .
\end{gathered}
$$

By putting Eq. (109) into Eq. (110), we obtain

$$
J_{2}=\frac{-T_{\mathrm{eq}}\left\langle\gamma_{U p}(\theta) \chi_{\mathrm{b}}(\theta)\right\rangle_{\theta}}{\frac{\left\langle\gamma_{p U}(\theta) \gamma_{U_{p}}(\theta)\right\rangle_{\theta}}{\gamma_{U U}}-\gamma_{p p}} F_{1}+\left\{G T_{\mathrm{eq}}^{2}\left\langle\chi_{\mathrm{b}}(\theta) \chi_{\mathrm{t}}(\theta)\right\rangle_{\theta}+\frac{\left\langle\gamma_{U p}(\theta) \chi_{\mathrm{b}}(\theta)\right\rangle_{\theta}\left\langle\gamma_{p U}(\theta) \chi_{\mathrm{b}}(\theta)\right\rangle_{\theta}}{\frac{\left\langle\gamma_{p U}(\theta) \gamma_{U p}(\theta)\right\rangle_{\theta}}{\gamma_{U U}}-\gamma_{p p}}\right\} F_{2} .
$$

Finally, from Eqs. (109) and (111), the quasilinear response coefficients are found to be

$$
L_{i j}^{\prime}=\left(\begin{array}{cc}
L_{11}^{\prime} & L_{12}^{\prime} \\
L_{21}^{\prime} & L_{22}^{\prime}
\end{array}\right)=\left(\begin{array}{cc}
-\frac{T_{\mathrm{eq}}^{2}}{\frac{\left\langle\gamma_{p U}(\theta) \gamma_{U p}(\theta)\right\rangle_{\theta}}{\gamma_{U U}}-\gamma_{p p}} & \frac{T_{\mathrm{eq}}\left\langle\gamma_{p U}(\theta) \chi_{\mathrm{b}}(\theta)\right\rangle_{\theta}}{\frac{\left\langle\gamma_{p U}(\theta) \gamma_{U p}(\theta)\right\rangle_{\theta}}{\gamma_{U U}}-\gamma_{p p}} \\
\frac{-T_{\mathrm{eq}}\left\langle\gamma_{U p}(\theta) \chi_{\mathrm{b}}(\theta)\right\rangle_{\theta}}{\frac{\left\langle\gamma_{p U}(\theta) \gamma_{U} p^{(\theta)}\right\rangle_{\theta}}{\gamma_{U U}}-\gamma_{p p}} & G T_{\mathrm{eq}}^{2}\left\langle\chi_{\mathrm{b}}(\theta) \chi_{\mathrm{t}}(\theta)\right\rangle_{\theta}+\frac{\left\langle\gamma_{U p}(\theta) \chi_{\mathrm{b}}(\theta)\right\rangle_{\theta}\left\langle\gamma_{p U}(\theta) \chi_{\mathrm{b}}(\theta)\right\rangle_{\theta}}{\frac{\left\langle\gamma_{p U}(\theta) \gamma_{U}(\theta)\right\rangle_{\theta}}{\gamma_{U U}}-\gamma_{p p}}
\end{array}\right),
$$


which are given using the phase averages $\langle\cdots\rangle_{\theta}$ of the quantities that include the Onsager kinetic coefficients using $\theta$ instead of $\theta_{\text {eq. }}$. By performing the phase averages in Eq. (112), we can confirm that Eq. (112) agrees with Eq. (69). From the expression of Eq. (112), we immediately note that the symmetric relation $L_{12}^{\prime}=L_{21}^{\prime}$ in the adiabatically eliminated model holds as a consequence of the antireciprocal relation of the Onsager kinetic coefficients $\gamma_{p U}(\theta)=-\gamma_{U p}(\theta)$ included in the three-dimensional dynamical model before the adiabatic elimination. Recalling that the antireciprocity of the Onsager kinetic coefficients reflects the time-reversal symmetry of the underlying microscopic dynamics [27], the present symmetric relation may also be attributed to the time-reversal symmetry. Although the antireciprocal terms do not contribute to the instantaneous entropy production rate, Eq. (100), during the relaxation dynamics, they can contribute to the entropy production rate averaged over one cycle period for the rotational state in the quasilinear response regime through $L_{i j}^{\prime}$ [Eq. (55)]. Interestingly, the restrictions on $L_{i j}^{\prime}$ in Eq. (56) imposed by the second law of thermodynamics are also assured by this antireciprocity.

\section{DISCUSSION AND SUMMARY}

This paper has presented the nonequilibrium thermodynamics of a nonlinear dynamics model of an LTD kinematic Stirling heat engine [6]. The two-dimensional dynamical equations describing the crank of the engine were derived from the original three-dimensional dynamical equations based on the adiabatic elimination of the gas dynamics. By using the two-dimensional dynamical equations, we investigated the stationary and rotational states, which are the fixed points and stable limit cycle of the equations, respectively. In particular, we focused on the regime near the bifurcation points and the quasilinear response regime sufficiently far from the bifurcation points of the latter state. The formal analytical expressions of the averaged angular velocity and heat fluxes (thermodynamic fluxes) as a function of the temperature difference and load torque (thermodynamic forces) were derived to explain these regimes in the rotational state. In the quasilinear response regime, it was found that the thermodynamic fluxes and forces are described by the linear relations with symmetric coefficients. Based on the linear relations, we obtained the maximum efficiency formula in terms of the coupling-strength parameter as the single figure of merit. We also demonstrated that the symmetric coefficients are considered to be a consequence of the antireciprocal relation of the Onsager kinetic coefficients in the relaxation dynamics before the adiabatic elimination.

Irrespective of whether the engine operates with external agents, such as conventional heat engines, or autonomously, such as in the present model, we analyzed their thermodynamic performance on an equal footing based on the linear relations [9-11]. When the adiabatic elimination is not valid, the dynamics of the gas and piston-crank system are not separated, and they constitute a dynamical system as a whole. When the adiabatic elimination is valid, the dynamics of the gas are completely subject to those of the piston-crank system. This yields explicit separation between the system and external agents. In this sense, there may not be much difference between conventional periodically driven heat engines operated by external agents and the present LTD kinematic Stirling engine, although the dynamics of the external operator itself in the latter case obeys the equations of motion. However, for the present self-sustained engine we have observed that the emergence of the symmetric coefficients can be explained based on the property of the relaxation dynamics towards the equilibrium state before the adiabatic elimination. This demonstrates the importance of modeling an autonomous heat engine as a dynamical system with mechanical and thermodynamic degrees of freedom. The symmetric relation can be experimentally verified in principle. It is of interest to investigate the similarities and differences between the present emergent symmetry in the quasilinear response regime and other various symmetries found in periodically driven heat engines operated by external agents in the linear response regime [12-17].

Our theory may be useful for predicting the possible future design of efficient LTD Stirling heat engines by employing our maximum efficiency formula. In this sense, although our theory is expected to describe existing LTD Stirling heat engines, it could also be used to describe more advanced engines in the near-future.

\section{ACKNOWLEDGMENTS}

The author is grateful to S. Toyabe for his insightful discussions. This work was supported by JSPS KAKENHI Grants No. 16K17765 and No. 19K03651.

\section{APPENDIX A: DERIVATION OF THE ADIABATIC APPROXIMATION SOLUTION, EQ. (17)}

Here, we derive the adiabatic approximation solution Eq. (17) based on [25]. By defining $T=T_{\text {eq }}+\delta T$, we can obtain the equation of $\delta T$ instead of $T$ from Eq. (13) as

$$
\frac{d \delta T}{d t}=-\frac{2 G}{f n R} \delta T-\frac{2}{f} \frac{d}{d t}[\ln V(\theta(t))] \delta T+X(\theta),
$$

where $X(\theta)$ is the external forcing exhibited by the crank, which is defined as

$$
X(\theta) \equiv \frac{2 G}{f n R} \frac{\sin \theta}{2} \Delta T-\frac{2 T_{\mathrm{eq}}}{f} \frac{d}{d t}[\ln V(\theta(t))] .
$$

Noting that Eq. (A1) is linear in $\delta T$, we can formally solve it as

$$
\delta T(t)=\int_{-\infty}^{t} X\left(\theta\left(t^{\prime}\right)\right) \exp \left[-\frac{2 G}{f n R}\left(t-t^{\prime}\right)-\frac{2}{f} \ln \frac{V(\theta(t))}{V\left(\theta\left(t^{\prime}\right)\right)}\right] d t^{\prime},
$$

where we have set $\delta T\left(t_{0}\right)=0$ with $t_{0}=-\infty$ as the initial condition because we are interested in the dynamics after the transient one. By integrating Eq. (A3) by parts, we have

$$
\begin{aligned}
\delta T(t)= & \frac{X(\theta(t))}{\frac{2 G}{f n R}+\frac{2}{f} \frac{d}{d t}[\ln V(\theta(t))]}-\int_{-\infty}^{t} \exp \left[-\frac{2 G}{f n R}\left(t-t^{\prime}\right)\right. \\
& \left.-\frac{2}{f} \ln \frac{V(\theta(t))}{V\left(\theta\left(t^{\prime}\right)\right)}\right] \frac{d}{d t^{\prime}}\left[\frac{X\left(\theta\left(t^{\prime}\right)\right)}{\frac{2 G}{f n R}+\frac{2}{f} \frac{d}{d t}\left[\ln V\left(\theta\left(t^{\prime}\right)\right)\right]}\right] d t^{\prime} .
\end{aligned}
$$


Equation (A4) is composed of the instantaneous (first term) and noninstantaneous (second term) response terms. If we can neglect the second term, the obtained solution $T=T_{\mathrm{eq}}+\delta T$ constitutes the adiabatic approximation solution in Eq. (17).

Let us consider a condition such that the second term in Eq. (A4) can be neglected compared to the first term. The absolute value of the second term in Eq. (A4) is bounded from the upper side as follows:

$$
\begin{aligned}
\mid \frac{d}{d t} & {\left.\left[\frac{X(\theta(t))}{\frac{2 G}{f n R}+\frac{2}{f} \frac{d}{d t}[\ln V(\theta(t))]}\right]\right|_{\max } } \\
& \times\left|\exp \left(-\frac{2}{f} \ln \frac{V(\theta(t))}{V\left(\theta\left(t^{\prime}\right)\right)}\right)\right| \int_{\max } \int_{-\infty}^{t} \exp \left[-\frac{2 G}{f n R}\left(t-t^{\prime}\right)\right] d t^{\prime} \\
= & \left|\frac{d}{d t}\left[\frac{X(\theta(t))}{\frac{2 G}{f n R}+\frac{2}{f} \frac{d}{d t}[\ln V(\theta(t))]}\right]\right|_{\max } \\
& \times\left|\exp \left(-\frac{2}{f} \ln \frac{V(\theta(t))}{V\left(\theta\left(t^{\prime}\right)\right)}\right)\right| \frac{f n R}{\max } .
\end{aligned}
$$

Because of $\left|\exp \left(-\frac{2}{f} \ln \frac{V(\theta(t))}{V\left(\theta\left(t^{\prime}\right)\right)}\right)\right|_{\max } \approx 1$, we can obtain the following condition such that the first term in Eq. (A4) is dominant:

$$
\begin{aligned}
\mid \frac{d}{d t} & {\left.\left[\frac{X(\theta(t))}{\frac{2 G}{f n R}+\frac{2}{f} \frac{d}{d t}[\ln V(\theta(t))]}\right]\right|_{\max } } \\
& \ll \frac{1}{\frac{f n R}{2 G}}\left|\frac{X(\theta(t))}{\frac{2 G}{f n R}+\frac{2}{f} \frac{d}{d t}[\ln V(\theta(t))]}\right| .
\end{aligned}
$$

This condition states that the time scale of the variation of the external forcing due to the crank is much longer than the system's intrinsic time scale.

\section{APPENDIX B: COMPARISON BETWEEN THE TWO-DIMENSIONAL DYNAMICAL MODEL AND THE THREE-DIMENSIONAL DYNAMICAL MODEL}

We compare the two-dimensional dynamical model, Eqs. (18) and (19), and the three-dimensional dynamical model, Eqs. (14)-(16). In Fig. 8, we show the $\langle\tilde{\omega}\rangle-\tilde{T}_{\text {load }}$ curve obtained by these two models for the two values $\tilde{G}=1.5$ [Fig. 8(a)] and $\tilde{G}=0.3$ [Fig. 8(b)]. For the numerical calculations, we used the nondimensionalized equations in Eqs. (18) and (19) for the two-dimensional dynamical model. For the three-dimensional dynamical model, we used the following nondimensionalized equations:

$$
\begin{gathered}
\frac{d \theta}{d \tilde{t}}=\tilde{\omega}, \\
\frac{d \tilde{\omega}}{d \tilde{t}}=\tilde{\sigma}\left(\frac{\tilde{T}}{\tilde{V}(\theta)}-\tilde{p}_{\mathrm{air}}\right) \sin \theta-\tilde{\Gamma} \tilde{\omega}-\tilde{T}_{\mathrm{load}}, \\
\frac{d \tilde{T}}{d \tilde{t}}=\frac{2}{f} \tilde{G}\left(\tilde{T}_{\mathrm{eff}}(\theta)-\tilde{T}\right)-\frac{2 \tilde{\sigma} \tilde{T} \sin \theta}{f \tilde{V}(\theta)} \tilde{\omega} .
\end{gathered}
$$

We find a good agreement between these two models for $\tilde{G}=1.5$, showing the validity of the adiabatic approximation, while for $\tilde{G}=0.3$ there is discrepancy between these two
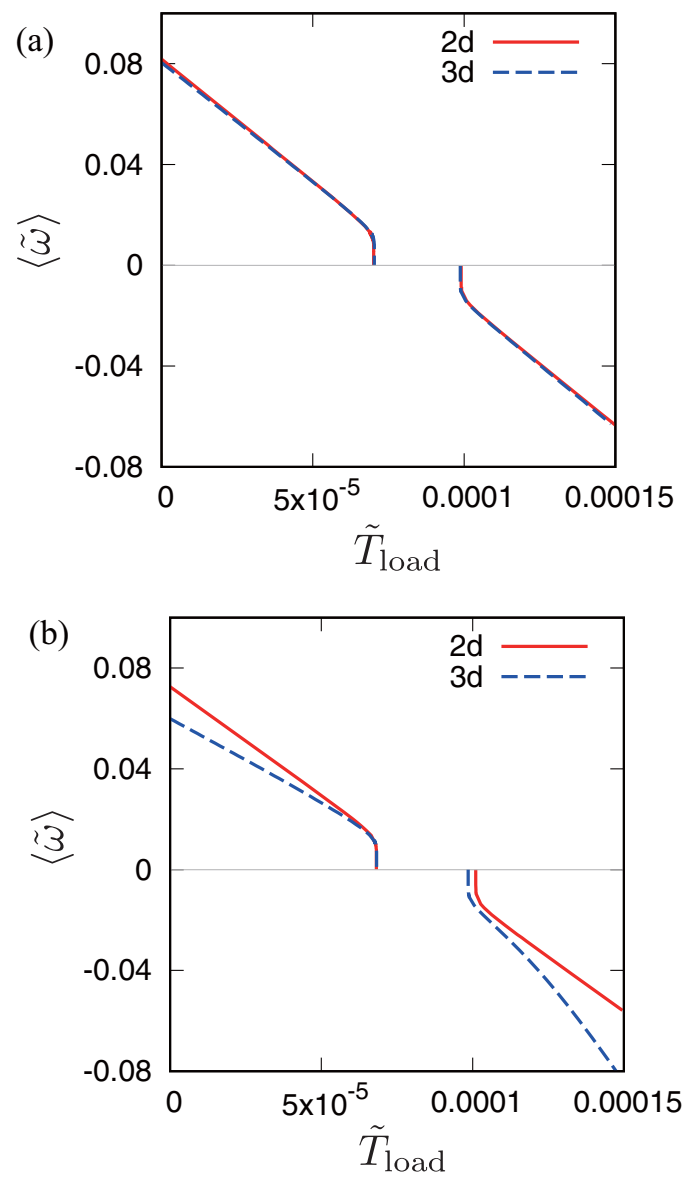

FIG. 8. $\langle\tilde{\omega}\rangle-\tilde{T}_{\text {load }}$ curve of the stable limit cycle for $\Delta \tilde{T}=$ 1/29.3. Equations (18) and (19) and Eqs. (14)-(16) are compared for two values: (a) $\tilde{G}=1.5$ and (b) $\tilde{G}=0.3$. We used $f=5$, and all the other parameters are the same as those in Fig. 3.

models. In particular, the three-dimensional dynamical model for $\tilde{G}=0.3$ shows an asymmetric behavior for the positive and negative rotational directions. Thus, the linear dependency observed for the two-dimensional dynamical model [see Fig. 3(a) and Eq. (44)] is not generally expected to hold in the three-dimensional case when the adiabatic approximation is not valid.

\section{APPENDIX C: DERIVATION OF EQ. (61) BASED ON THE ENERGY CONSERVATION LAW}

Here, we show Eq. (61) from the energy conservation law $P_{\text {load }}=\left\langle J_{Q_{b}}\right\rangle+\left\langle J_{Q_{\mathrm{t}}}\right\rangle-P_{\text {fric }}$. We need the nonlinear terms of $\Delta \tilde{T} \tilde{\omega}$ and $\tilde{\omega}^{2}$ that were neglected in Eq. (37):

$$
\begin{aligned}
T(\theta, \omega) \simeq & T_{\text {eff }}(\theta)-T_{\mathrm{eq}}\left(1+\frac{\sin \theta}{2} \Delta \tilde{T}\right) \frac{r \sin \theta \sigma_{\mathrm{p}}}{\tilde{G} V(\theta)} \tilde{\omega} \\
& +T_{\mathrm{eq}} \frac{r^{2} \sin ^{2} \theta \sigma_{\mathrm{p}}^{2}}{\tilde{G}^{2} V^{2}(\theta)} \tilde{\omega}^{2} .
\end{aligned}
$$


By substituting Eq. (C1) into Eq. (6) and time-averaging, we can approximate $\left\langle J_{Q_{b}}\right\rangle$ and $\left\langle J_{Q_{t}}\right\rangle$ as

$$
\begin{aligned}
&\left\langle J_{Q_{\mathrm{b}}}\right\rangle \simeq \frac{G}{8} \Delta T+\frac{T_{\mathrm{eq}} n R r \sigma_{\mathrm{p}}}{2}\left\langle\frac{\sin ^{2} \theta}{V(\theta)}\right\rangle_{\theta} \Omega \\
&+\frac{n R r \sigma_{\mathrm{p}}}{2}\left\langle\frac{\sin ^{2} \theta}{V(\theta)}\right\rangle_{\theta} \Delta T \Omega-\frac{T_{\mathrm{eq}} n^{2} R^{2} r^{2} \sigma_{\mathrm{p}}^{2}}{2 G}\left\langle\frac{\sin ^{2} \theta}{V^{2}(\theta)}\right\rangle_{\theta} \Omega^{2}, \\
&\left\langle J_{Q_{\mathrm{t}}}\right\rangle \simeq-\frac{G}{8} \Delta T-\frac{T_{\mathrm{eq}} n R r \sigma_{\mathrm{p}}}{2}\left\langle\frac{\sin ^{2} \theta}{V(\theta)}\right\rangle_{\theta} \Omega \\
&-\frac{T_{\mathrm{eq}} n^{2} R^{2} r^{2} \sigma_{\mathrm{p}}^{2}}{2 G}\left\langle\frac{\sin ^{2} \theta}{V^{2}(\theta)}\right\rangle_{\theta} \Omega^{2},
\end{aligned}
$$

including the nonlinear terms. Therefore, we have

$$
\begin{aligned}
\left\langle J_{Q_{\mathrm{b}}}\right\rangle+\left\langle J_{Q_{\mathrm{t}}}\right\rangle-P_{\text {fric }}= & \frac{n R r \sigma_{\mathrm{p}}}{2}\left\langle\frac{\sin ^{2} \theta}{V(\theta)}\right\rangle_{\theta} \Delta T \Omega \\
& -\frac{T_{\mathrm{eq}} n^{2} R^{2} r^{2} \sigma_{\mathrm{p}}^{2}}{G}\left\langle\frac{\sin ^{2} \theta}{V^{2}(\theta)}\right\rangle_{\theta} \Omega^{2}-\Gamma \Omega^{2} \\
= & \frac{L_{12}^{\prime}}{L_{11}^{\prime}} J_{1} F_{2} T_{\mathrm{eq}}-\frac{T_{\mathrm{eq}}}{L_{11}^{\prime}} J_{1}^{2} \\
= & P_{\text {load }} .
\end{aligned}
$$

We note that $\left\langle J_{Q_{\mathrm{t}}}\right\rangle=-\left\langle J_{Q_{b}}\right\rangle$ up to the linear order of $\Delta T$ and $T_{\text {load }}$ in Eqs. (C2) and (C3). Thus, the nonlinear terms are found to play an important role in energetics, though they do not appear in the linear relations Eqs. (44) and (45) in the quasilinear response regime.
[1] J. R. Senft, An Introduction to Stirling Engines, 8th ed. (Moriya Press/Open Library, Wis., 2010).

[2] B. Kongtragool and S. Wongwises, A review of solar-powered Stirling engines and low temperature differential Stirling engines, Renew. Sustain. Energy Rev. 7, 131 (2003).

[3] J. R. Senft, An Introduction to Low Temperature Differential Stirling Engines, 4th ed. (Moriya Press/Open Library, Wis., 2000).

[4] A. Robson, T. Grassie, and J. Kubie, Modelling of a lowtemperature differential Stirling engine, Proc. IMechE Part C: J. Mech. Eng. Sci. 221, 927 (2007).

[5] M. Craun and B. J. Bamieh, Control-oriented modeling of the dynamics of Stirling engine regenerators, Dynam. Syst. Meas., Control 140, 041001 (2018).

[6] Y. Izumida, Nonlinear dynamics analysis of a low-temperaturedifferential kinematic Stirling heat engine, Europhys. Lett. 121, 50004 (2018).

[7] S. H. Strogatz, Nonlinear Dynamics and Chaos: With Applications to Physics, Biology, Chemistry, and Engineering (Westview Press, Boulder, CO, 2001).

[8] S. Toyabe and Y. Izumida, Experimental characterization of autonomous heat engine based on minimal dynamical-system model, arXiv:1911.02810v3.

[9] C. Van den Broeck, Thermodynamic Efficiency at Maximum Power, Phys. Rev. Lett. 95, 190602 (2005).

[10] B. Jiménez de Cisneros and A. Calvo Hernández, Collective Working Regimes for Coupled Heat Engines, Phys. Rev. Lett. 98, 130602 (2007).

[11] B. Jiménez de Cisneros and A. Calvo Hernández, Coupled heat devices in linear irreversible thermodynamics, Phys. Rev. E 77, 041127 (2008).

[12] Y. Izumida and K. Okuda, Onsager coefficients of a finite-time Carnot cycle, Phys. Rev. E 80, 021121 (2009).

[13] Y. Izumida and K. Okuda, Onsager coefficients of a Brownian Carnot cycle, Eur. Phys. J. B 77, 499 (2010).

[14] Y. Izumida and K. Okuda, Linear irreversible heat engines based on local equilibrium assumptions, New J. Phys. 17, 085011 (2015).

[15] K. Brandner, K. Saito, and U. Seifert, Thermodynamics of Nicro- and Nano-Systems Driven by Periodic
Temperature Variations, Phys. Rev. X 5, 031019 (2015).

[16] K. Proesmans and C. Van den Broeck, Onsager Coefficients in Periodically Driven Systems, Phys. Rev. Lett. 115, 090601 (2015).

[17] L. Cerino, A. Puglisi, and A. Vulpiani, Linear and nonlinear thermodynamics of a kinetic heat engine with fast transformations, Phys. Rev. E 93, 042116 (2016).

[18] F. Curzon and B. Ahlborn, Efficiency of a Carnot engine at maximum power output, Am. J. Phys. 43, 22 (1975).

[19] P. Salamon, J. D. Nulton, G. Siragusa, T. R. Anderse, and A. Limon, Principles of control thermodynamics, Energy 26, 307 (2001).

[20] R. S. Berry, V. A. Kazakov, S. Sieniutycz, Z. Szwast, and A. M. Tsirlin, Thermodynamics Optimization of Finite-Time Processes (Wiley, Chichester, UK, 2000).

[21] L. Onsager, Reciprocal relations in irreversible processes. I, Phys. Rev. 37, 405 (1931).

[22] H. B. Callen, Principle of minimum entropy production, Phys. Rev. 105, 360 (1957).

[23] Y. J. Lu, H. Nakahara, and J. S. Bobowski, Quantitative Stirling cycle measurements: $P-V$ diagram and refrigeration, Phys. Teach. 58, 18 (2020).

[24] A. Medina, P. L. Curto-Risso, A. C. Hernández, L. GuzmánVargas, F. Angulo-Brown, and A. K. Sen, Quasi-dimensional Simulation of Spark Ignition Engines: From Thermodynamic Optimization to Cyclic Variability (Springer, London, 2014).

[25] H. Haken, Synergetics, An Introduction: Nonequilibrium Phase Transitions and Self-Organization in Physics, Chemistry and Biology, 2nd ed. (Springer, Berlin, 1978).

[26] J. Chen, Z. Yan, G. Lin, and B. Andresen, On the CurzonAhlborn efficiency and its connection with the efficiencies of real heat engines, Energy Convers. Manage. 42, 173 (2001).

[27] L. D. Landau and E. M. Lifshitz, Course of Theoretical Physics, Vol. 5. Statistical Physics, 3rd ed. (Elsevier, Amsterdam, 1980), Part 1.

[28] T. Heimburg, Linear nonequilibrium thermodynamics of reversible periodic processes and chemical oscillations, Phys. Chem. Chem. Phys. 19, 17331 (2017). 